\title{
Littlewood-Paley-Stein theory for semigroups in UMD spaces
}

Tuomas P. Hytönen

\begin{abstract}
The Littlewood-Paley theory for a symmetric diffusion semigroup $T^{t}$, as developed by Stein, is here generalized to deal with the tensor extensions of these operators on the Bochner spaces $L^{p}(\mu, X)$, where $X$ is a Banach space. The $g$-functions in this situation are formulated as expectations of vector-valued stochastic integrals with respect to a Brownian motion. A two-sided $g$-function estimate is then shown to be equivalent to the UMD property of $X$. As in the classical context, such estimates are used to prove the boundedness of various operators derived from the semigroup $T^{t}$, such as the imaginary powers of the generator.
\end{abstract}

\section{Introduction}

The aim of this paper is to obtain a vector-valued extension of the general Littlewood-Paley theory as developed by E. M. Stein in his 1970 monograph [21]. We consider a one-parameter family $\left(T^{t}\right)_{t>0}$, which we call a symmetric diffusion semigroup on a $\sigma$-finite measure space $(M, \mu)$ provided that each $T^{t}$ is a linear mapping on $\bigcup_{p \in[1, \infty]} L^{p}(\mu)$, which satisfies

- $T^{t+s}=T^{t} T^{s}$ for all $0<s, t<\infty$ (semigroup property),

- $T^{t} f \rightarrow f$ in $L^{2}(\mu)$ as $t \downarrow 0$ for $f \in L^{2}(\mu)$ (continuity),

and in addition each $T^{t}$ is a bi-stochastic operator. This last condition entails the following:

- $\left\|T^{t} f\right\|_{L^{p}(\mu)} \leq\|f\|_{L^{p}(\mu)}$ for $1 \leq p \leq \infty$ (contractiveness),

- $T^{t}$ is self-adjoint on $L^{2}(\mu)$ (symmetry),

2000 Mathematics Subject Classification: 42A61 (Primary); 42B25, 46B09, 46B20 (Secondary).

Keywords: Brownian motion, diffusion semigroup, functional calculus, stochastic integral, unconditional martingale differences. 
- $T^{t} f \geq 0$ if $f \geq 0$ (positivity),

- $\int_{M} T^{t} f \mathrm{~d} \mu=\int_{M} f \mathrm{~d} \mu$ for all $f \in L^{1}(\mu)$ (conservativeness).

Typical examples are the Gauss and Poisson semigroups $e^{t \triangle}$ and $e^{-t(-\Delta)^{1 / 2}}$ on the unit-circle or on $\mathbb{R}^{n}$, where $\triangle$ is the corresponding Laplace operator. The semigroup $e^{-t(-\triangle)^{1 / 2}}$ is the one originally considered by J. Littlewood and R.E. A. C. Paley, and it has the important additional property of being "subordinated" by another symmetric diffusion semigroup, namely $e^{t \Delta}$, in the sense that

$$
e^{-t(-\triangle)^{1 / 2}} f=\frac{1}{\sqrt{\pi}} \int_{0}^{\infty} \frac{e^{-u}}{\sqrt{u}} e^{t^{2} \triangle / 4 u} f \mathrm{~d} u .
$$

More generally, if $T^{t}=e^{-t A}$ is a symmetric diffusion semigroup with generator $-A$, and $P^{t}=e^{-t A^{1 / 2}}$ (in which case the above integral representation holds with $-A$ in place of $\triangle$ ), we say that $P^{t}$ is the subordinated semigroup of $T^{t}$.

Thanks to positivity, these semigroups have tensor extensions to the Bochner spaces $L^{p}(\mu, X)$, where $X$ is an arbitrary Banach space. They satisfy the same properties listed above when these are meaningful, and hence we do not make a notational distinction between $T^{t}$ on $L^{p}(\mu)$ and its tensor extension on $L^{p}(\mu, X)$, which is the key object of our study.

A central rôle in the classical Littlewood-Paley theory is played by various $g$-functions, or square-functions, of which typical examples are

$$
g_{k}(f)(x)=\left(\int_{0}^{\infty}\left|t^{k} \frac{\partial^{k}}{\partial t^{k}} T^{t} f(x)\right|^{2} \frac{\mathrm{d} t}{t}\right)^{1 / 2}
$$

where $f \in L^{p}(\mu), x \in M$, and $k=1,2, \ldots$

The principal motivation for introducing these objects is to provide new equivalent norms for the spaces $L^{p}(\mu)$, which make the boundedness properties of various operators derived from the semigroup $T^{t}$ more transparent. In fact, it is shown in [21] that under the above assumptions we have

$$
c\left\|f-E_{0} f\right\|_{L^{p}(\mu)} \leq\left\|g_{k}(f)\right\|_{L^{p}(\mu)} \leq C\|f\|_{L^{p}(\mu)},
$$

for all $f \in L^{p}(\mu), 1<p<\infty$, and $k=1,2, \ldots$, where $E_{0} f=\lim _{t \rightarrow \infty} T^{t} f$ is the projection onto the fixed point space of $\left(T^{t}\right)_{t>0}$ and $0<c<C<\infty$ only depend on $p$ and $k$. The existence of the mentioned limit for $f \in L^{2}(\mu)$ follows from Hilbert space spectral theory, whereas for $\left.f \in L^{p}(\mu), p \in\right] 1, \infty[$, it is a consequence of the density of $L^{2}(\mu) \cap L^{p}(\mu)$ and the maximal estimate (see [21, §III.3]) $\left\|\sup _{0<t<\infty}\left|T^{t} f\right|\right\|_{L^{p}(\mu)} \leq C\|f\|_{L^{p}(\mu)}$. Since $E_{0}$ is a positive operator, it has a bounded tensor extension to $L^{p}(\mu, X)$ for $\left.p \in\right] 1, \infty[$, too, and this extension is again seen to be the strong limit as $t \rightarrow \infty$ of the tensor extensions $T^{t}$. 
As an application of the two-sided inequality (1.1), it is relatively easy to obtain the $L^{p}(\mu)$ boundedness of the "multiplier" operators

$$
T_{a} f=-\int_{0}^{\infty} a(t) \frac{\partial}{\partial t} T^{t} f \mathrm{~d} t
$$

where $a \in L^{\infty}(0, \infty)$. This follows from the point-wise estimate

$$
g_{1}\left(T_{a} f\right)(x) \leq\|a\|_{\infty} g_{2}(f)(x)
$$

and the observation that $E_{0} T_{a}=0$. When $T^{t}$ is the Poisson semigroup, the operators $T_{a}$ include e.g. the imaginary powers $(-\triangle)^{\text {is }}$ of the Laplacian with $a(t)=\Gamma(1-\mathbf{i} s)^{-1} t^{-\mathbf{i} s}$.

One can obviously think of several different ways of making a "vectorvalued generalization" of a given classical result. The most immediate is to replace all absolute values by norms of a Banach space $X$ and try to prove the resulting vector-valued statement, possibly under some geometric conditions on the space $X$. Such an extension has been obtained for the LittlewoodPaley theory of $e^{-t(-\triangle)^{1 / 2}}$ by Q. Xu [23] (where another kind of extension is also given for Banach lattices, commented on below), and very recently for the general Littlewood-Paley-Stein theory by T. Martínez, J. L. Torrea and $\mathrm{Xu}$ [14]. They show that the left (respectively, right) side of (1.1) with $k=1$ continues to hold in $L^{p}(\mu, X)$ for every subordinated semigroup $T^{t}$ if and only if $X$ has martingale type 2 (resp., martingale cotype 2). (We refer to the mentioned papers for the definition of these notions, which we shall not use outside this introductory discussion.) More generally, with

$$
\mathfrak{G}_{q}(f)(x):=\left(\int_{0}^{\infty}\left|t \frac{\partial}{\partial t} P^{t} f(x)\right|_{X}^{q} \frac{\mathrm{d} t}{t}\right)^{1 / q}
$$

they prove the following:

1.3 Theorem ([14, 23]). Let $X$ be a Banach space, and $1<q, p<\infty$. Then $X$ has martingale type $q$ (resp. martingale cotype $q$ ), if and only if the following estimate is true for every subordinated semigroup $P^{t}$ on some $(M, \mu)$, if and only if it is true for the Poisson semigroup on the unit-circle:

$$
c\left\|f-E_{0} f\right\|_{L^{p}(\mu, X)} \leq\left\|\mathfrak{G}_{q} f\right\|_{L^{p}(\mu)} \quad\left(\text { resp. }\left\|\mathfrak{G}_{q} f\right\|_{L^{p}(\mu)} \leq C\|f\|_{L^{p}(\mu, X)}\right)
$$

for all $f \in L^{p}(\mu, X)$.

Note that the martingale type (resp. cotype) $q$ of any Banach space necessarily satisfies $q \leq 2$ (resp. $q \geq 2$ ). Thus the only way that a twosided estimate can hold in Theorem 1.3 is to have $q=2$ and $X$ having 
both martingale type and cotype 2 , which in turn is equivalent to $X$ being (isomorphic to) a Hilbert space. This is not at all untypical of vector-valued extensions of classical orthogonality estimates.

In this paper, we generalize the results in [21] along a different line, which yields a $g$-function characterization of another class of Banach spaces. To this end, we employ the familiar method of "randomization", a continuous version of which is here required. This is provided by the Itô isometry for stochastic integrals with respect to a Brownian motion $\left(B_{t}\right)_{t \geq 0}$ :

$$
\left(\int_{0}^{\infty}|f(t)|_{H}^{2} \mathrm{~d} t\right)^{1 / 2}=\left(E\left|\int_{0}^{\infty} f(t) \mathrm{d} B_{t}\right|_{H}^{2}\right)^{1 / 2}
$$

where $H$ is a Hilbert space and $E$ is the expectation on the probability space $(\Omega, P)$ supporting the Brownian motion. While the two sides of (1.4) are incomparable if $H$ is replaced by a general Banach space $X$, the success of the discrete randomization procedure in vector-valued harmonic analysis (see e.g. $[4,5,13,22]$ ) suggests that the right side of (1.4) might be better suited for generalization of the two-sided estimates of Littlewood-PaleyStein theory beyond Hilbert spaces. The required theory of stochastic integration of functions with values in an arbitrary Banach space was initiated by J. Rosiński and Z. Suchanecki [17], and it has been extended and developed in the recent work of J. M. A. M. van Neerven and L. Weis [16]. The idea of using the stochastic square-functions, in turn, is due to N. Kalton and Weis [12], who formulate them in different but equivalent terms, however.

The geometric condition for a vector-valued extension of the "stochastic" Littlewood-Paley-Stein theory turns out to be the property of unconditionality of martingale differences (UMD), which is not surprising in view of the rôle of the UMD class of Banach spaces as the ultimate setting to which several results in Calderón-Zygmund, Fourier multiplier, and stochastic integration theory can be extended; cf. $[4,5,13,15,22]$ and further references there.

Recall that $X$ is a UMD space if for some (equivalently, all) $p \in] 1, \infty[$ there is a constant $C<\infty$ such that

$$
\left\|\sum_{k=1}^{n} \epsilon_{k} d_{k}\right\|_{L^{p}(\mu, X)} \leq C\left\|\sum_{k=1}^{n} d_{k}\right\|_{L^{p}(\mu, X)},
$$

whenever $\left(d_{k}\right)_{k=1}^{n} \in L^{p}(\mu, X)^{n}$ is a martingale difference sequence and $\left(\epsilon_{k}\right)_{k=1}^{n} \in$ $\{-1,+1\}^{n}$. We refer to [19] for a survey of UMD spaces.

The main result of this paper is the following: 
1.6 Theorem. Let $X$ be Banach space. If $X$ is $U M D$ and $P^{t}$ is a subordinated semigroup on some $(M, \mu)$, then the stochastic integrals below exist and satisfy

$$
c\left\|f-E_{0} f\right\|_{L^{p}(\mu, X)} \leq E\left\|\int_{0}^{\infty} t^{k} \frac{\partial^{k}}{\partial t^{k}} P^{t} f \frac{\mathrm{d} B_{t}}{t^{1 / 2}}\right\|_{L^{p}(\mu, X)} \leq C\|f\|_{L^{p}(\mu, X)}
$$

for all $\left.f \in L^{p}(\mu, X), p \in\right] 1, \infty[$, and $k=1,2, \ldots$, where $0<c<C<\infty$ only depend on $X, p$ and $k$. Conversely, if (1.7) holds for $k=1$ and some $p \in] 1, \infty\left[\right.$ when $P^{t}$ is the subordinated Poisson semigroup on the unit-circle, then $X$ is a UMD space.

While the expression in the middle of (1.7) does not exactly coincide with $\left\|g_{k}(f)\right\|_{L^{p}(\mu, H)}$ for $X=H$, one can easily see their equivalence by two applications of the equivalence of different $L^{p}$ norms of a Gaussian random variable, Fubini's theorem and the Itô isometry; but we find it more convenient to work with the formulation above.

We also note that if $X$ is a Banach lattice of functions on a $\sigma$-finite measure space $(N, \nu)$, and $X$ has finite cotype (which every UMD space does), then the expectation of an $X$-valued stochastic integral admits an equivalent formulation more close in appearance to classical square functions (see [16], Cor. 2.10), so that (1.7) reads

$$
\begin{aligned}
c\left\|f-E_{0} f\right\|_{L^{p}(\mu, X)} & \leq\left[\int_{M}\left\|\left(\int_{0}^{\infty}\left|t^{k} \frac{\partial^{k}}{\partial t^{k}} P^{t} f(x, \cdot)\right|^{2} \frac{\mathrm{d} t}{t}\right)^{\frac{1}{2}}\right\|_{X}^{p} \mathrm{~d} \mu(x)\right]^{\frac{1}{p}} \\
& \leq C\|f\|_{L^{p}(\mu, X)},
\end{aligned}
$$

where $f \in L^{p}(\mu, X)$ is viewed as a two-variable function $f(x, y)$ on $M \times N$.

The equivalence of the UMD property of a Banach lattice $X$ to a twosided estimate like this was already established by $\mathrm{Xu}$ [23]. Related results in general Banach spaces have also been obtained by Kalton and Weis [12] in the framework of holomorphic functional calculus of Banach space operators. In contrast to this complex variable approach of [12], our methods (with the exception of the last two sections) are based on real variable techniques.

The proof of the square-function estimate from the UMD condition follows the method of Stein from [21] and its extension in [14]: The UMD property is used to prove a new inequality, Prop. 4.1, for $X$-valued martingales, which is then transferred by Rota's representation theorem into a weak Littlewood-Paley inequality, Theorem 5.1, for an arbitrary symmetric diffusion semigroup $T^{t}$. The fact that $P^{t}$ is subordinated by such a $T^{t}$ then allows to obtain the right estimate in (1.7) from the intermediate estimate 
for $T^{t}$. The left inequality, in turn, will follow from a duality argument and the fact that the right side is also satisfied in $L^{p^{\prime}}\left(\mu, X^{\prime}\right)$ by self-duality of the UMD property. Unfortunately, the method will not yield (1.7) for general symmetric diffusion semigroups $T^{t}$, but this can be achieved in a more restricted class of Banach spaces, as explained below.

The converse implication is based on a modification of S. Guerre-Delabrière's [11] proof of the fact that the $L^{p}(0,1 ; X)$ boundedness of the imaginary powers $(-\triangle)^{\text {is }}$ of the Laplacian on the unit-circle implies the UMD property for $X$; it has similarities with the proof of the converse part of Theorem 1.3 in [23]. Note that if we assumed (1.7) for both $k=1$ and $k=2$, then we could prove the boundedness of $(-\triangle)^{\mathbf{i} s}$ almost like in [21], and obtain the necessity of UMD directly from Guerre-Delabrière's theorem. Under the stated weaker hypothesis, a little more work is needed.

The two-sidedness of the square-function inequalities allows to use the equivalent randomized norms to the estimation of the "multiplier" transformations (1.2), and even their operator-valued versions, where $a:[0, \infty[\rightarrow$ $\mathscr{L}(X)$ is a strongly measurable function with $R$-bounded range. Recall that $R$-boundedness of a family $\mathscr{T} \subset \mathscr{L}(X)$ means the uniform estimate

$$
E\left|\sum_{k=1}^{n} \varepsilon_{k} T_{k} x_{k}\right|_{X} \leq C E\left|\sum_{k=1}^{n} \varepsilon_{k} x_{k}\right|_{X},
$$

which should hold for all $n \in \mathbb{Z}_{+}, x_{k} \in X$ and $T_{k} \in \mathscr{T}$, and for independent random variables $\varepsilon_{k}$ on some probability space $\Omega$ with distribution $P\left(\varepsilon_{k}=+1\right)=P\left(\varepsilon_{k}=-1\right)=1 / 2$. This notion, which was introduced by E. Berkson and T. A. Gillespie [1], has recently proved crucial in connection with Fourier multipliers and Calderón-Zygmund operators with operatorvalued symbols and kernels, cf. e.g. $[5,6,13,22]$. We denote by $\mathscr{R}(\mathscr{T})$ the $R$-bound of the set $\mathscr{T}$, i.e., the smallest admissible constant in (1.8), and for an operator-valued function we abbreviate $\mathscr{R}(a):=\mathscr{R}($ range $a)$. We shall prove the following operator-valued Marcinkiewicz-Stein multiplier theorem:

1.9 Theorem. Let $X$ be a UMD space, $p \in] 1, \infty\left[\right.$, and $P^{t}$ a subordinated semigroup on a measure space $(M, \mu)$. Let $a:\left[0, \infty\left[\rightarrow \mathscr{L}\left(L^{p}(\mu, X)\right)\right.\right.$ be a strongly measurable function such that

- the range of a is $R$-bounded, and

- $a(t)$ commutes with $P^{s}$ for all $0 \leq s, t<\infty$.

Then $T_{a}$ as in (1.2), with $P^{t}$ in place of $T^{t}$, defines a bounded operator on $L^{p}(\mu, X)$, with norm estimated by $C \mathscr{R}(a)$, where $C$ depends only on $X$ and $p$. 
This recovers and extends Stein's [21, Cor. 3, p. 121] for the case of subordinated semigroups, but does not contain the case of general symmetric diffusion semigroups, due to the same limitation as in Theorem 1.6. It also gives a partial extension of a result of P. Clément and J. Prüss [6, Cor. 3], who proved an operator-valued Marcinkiewicz-Stein multiplier theorem under the same assumptions on $a$, for more general (positive, analytic, and contractive) semigroups $P^{t}$, but requiring $X$ to be an $L^{q}(\nu)$ space, $\left.q \in\right] 1, \infty[$; the question which motivated the present investigation, whether $L^{q}(\nu)$ could be replaced by an arbitrary UMD space, was posed in [6].

That the UMD assumption cannot be weakened follows from GuerreDelabrière's theorem [11], since $(-\triangle)^{\text {is }}$ is covered by Theorem 1.9 when $P^{t}$ is the Poisson semigroup on the unit-circle, so that also Theorem 1.9 is actually a characterization of UMD spaces.

Let us mention that both Theorems 1.6 and 1.9 remain to hold (see Theorem 9.7 and Corollary 9.10) for all symmetric diffusion semigroups if we strengthen the geometric condition as follows: $X$ should be (isomorphic to) a closed subspace of a complex interpolation space $[H, Y]_{\theta}, 0<\theta<1$, where $H$ is a Hilbert space and $Y$ is another UMD space. In this situation one can obtain improved inequalities in $L^{p}(\mu, X)$ by interpolating between the weaker estimates derived from the UMD condition in $L^{q}(\mu, Y)$ and strong results guaranteed by Hilbert space spectral theory in $L^{2}(\mu, H)$, in much the way as in Stein's original treatise [21] when $X=Y=H=\mathbb{C}$.

The same phenomenon is present in the vector-valued extension of Martínez, Torrea and Xu [14]: Also in their case, the square-function estimates in Theorem 1.3 are shown to hold for all symmetric diffusion semigroups if $X$ is an appropriate interpolation space.

The rest of the paper is organized as follows: In $\S 2$ we present the required preliminaries from vector-valued stochastic integration. $\S 3$ is concerned with proving the converse part of Theorem 1.6, the necessity of UMD for $g$ function estimates. The other direction of Theorem 1.6 is handled in the next three sections: In $\S 4$ we prove a certain martingale inequality, which is transferred by Rota's theorem into a weak $g$-function estimate in $\S 5$. This, in turn, is used to prove the full $g$-function estimate of Theorem 1.6 for subordinated semigroups in $\S 6$. Theorem 1.9 is proved in $\S 7$, and in $\S 8$ we relate this result to the holomorphic functional calculus for the generator of the semigroup $P^{t}$. Finally, the interpolation results outlined above are detailed in $\S 9$.

Acknowledgement. This research was carried out during my one-year stay at the Technische Universiteit Delft in 2004-5, funded by the European research training network HPRN-CT-2002-00281 "Evolution equations for deterministic and stochastic systems". Most of the results and proofs of this 
paper were first presented in a series of lectures at the Delft seminar "Probabilistic methods in PDE's" during this period. The seminar was attended by a number of students and the Professors Philippe Clément and Jan van Neerven, whose questions and comments helped improve the final outcome substantially. I am particularly grateful to Jan van Neerven for discovering a gap in my earlier proof of Theorem 1.9. The results were also presented in talks at Universidad Autónoma de Madrid and Université de FrancheComté, and I wish to thank the interested audience in both occasions, and especially Professors José Luis Torrea and Quanhua Xu, and Dr. Teresa Martínez for helpful comments.

\section{Stochastic integration theory of van Neerven and Weis}

In this section we collect the required definitions and results from the Banach space-valued stochastic integration theory of van Neerven and Weis [16], which we shall extensively exploit in the sequel. While the slightly less general definition of such integrals by Rosiński and Suchanecki [17] would be sufficient for our purposes, an important rôle in our work is played by various estimates and convergence results for the stochastic integrals, which are due to van Neerven and Weis.

These authors work explicitly with functions on an interval $[0, T]$ and a Brownian motion $\left(B_{t}\right)_{t \in[0, T]}$, but it is pointed out in [16, Remark 2.2] that all their results hold just as well for an arbitrary finite measure space with a Gaussian random measure (see below), which is the framework of [17]. We would like to mention that even the $\sigma$-finite case, in which we shall be working, can be treated in essentially the same way. Below, we formulate the required results from [16] in a setting suitable for our needs, which differs slightly from the original statements; there is, however, very little effect on proofs.

2.1 Definition. Let $(M, \mathscr{M}, \mu)$ be a $\sigma$-finite measure space, and let $\mathscr{M}_{0}$ denote the subalgebra of $\mathscr{M}$ consisting of the sets of finite $\mu$-measure. A Gaussian random measure on $(M, \mathscr{M}, \mu)$ is a set function

$$
W=W_{\mu}: \mathscr{M}_{0} \rightarrow L^{2}(\Omega)
$$

where $(\Omega, \Sigma, P)$ is some probability space, having the following properties:

- For all $E \in \mathscr{M}_{0}$, the random variable $W(E)$ is centred Gaussian with variance $\mu(E)$.

- For all disjoint $E_{1}, \ldots, E_{n} \in \mathscr{M}_{0}$, the random variables $W\left(E_{k}\right)$ are independent and

$$
W\left(\bigcup_{k=1}^{n} E_{k}\right)=\sum_{k=1}^{n} W\left(E_{k}\right) .
$$


We shall mostly be concerned with $M=[0, \infty[$, but also with $M=$ $\left[0, \infty\left[^{2}\right.\right.$. These spaces are always understood to be equipped with the Borel $\sigma$-algebra and the Lebesgue measure. In the former case the associated Gaussian random measure is called a Brownian motion, for which we employ the conventional notation $B_{t}$, while in the latter it is a Brownian sheet.

Note that in the setting of Defintion 2.1, a Gaussian random measure always exists (provided that $(\Omega, \Sigma, P)$ is sufficiently rich): Let $\left(\phi_{k}\right)_{k=1}^{\infty}$ be an orthonormal basis of $L^{2}(\mu)$, and let $\left(\gamma_{k}\right)_{k=1}^{\infty}$ be an independent sequence of standard Gaussian variables on $\Omega$. Then $W(E):=\sum_{k=1}^{\infty} \gamma_{k} \int_{E} \phi_{k} \mathrm{~d} \mu$ is easily seen to satisfy the above properties.

For a simple function $f=\sum_{k=1}^{n} a_{k} 1_{E_{k}}$, where the $E_{k}$ are disjoint sets of finite measure, the stochastic integral is defined in the only reasonable way as

$$
\int_{M} f \mathrm{~d} W:=\sum_{k=1}^{n} a_{k} W\left(E_{k}\right) .
$$

If $f$ takes its values in a Hilbert space $H$, one easily verifies the Itô isometry $\left\|\int_{M} f \mathrm{~d} W\right\|_{L^{2}(\Omega, H)}=\|f\|_{L^{2}(\mu, H)}$, which immediately yields an extension of the stochastic integral to all $L^{2}(\mu, H)$ by density.

We now proceed to extend the notion of stochastic integration to general Banach spaces, following van Neerven and Weis. The theory of [16] is built for real spaces, which makes us adopt the following convention: By the dual $X^{\prime}$ of a Banach space $X$, we always understand $X^{\prime}:=\mathscr{L}(X, \mathbb{R})$. Clearly we can view any complex Banach space also as a real Banach space, where all the original operations are still defined; however, we must think of multiplication by a $z \in \mathbb{C} \backslash \mathbb{R}$ not as scalar multiplication but as a bounded linear operator on $X$.

2.2 Definition ([16]). Let $(M, \mathscr{M}, \mu)$ be a $\sigma$-finite measure space. If $X$ is a Banach space and $\phi: M \rightarrow X$ a function, we say that $\phi$ is weakly $L^{2}$ if $\left\langle\phi(\cdot), x^{\prime}\right\rangle \in L^{2}(\mu)$ for every $x^{\prime} \in X^{\prime}$.

Let $W$ be a Gaussian random measure on $(M, \mathscr{M}, \mu)$, its values being random variables on a probability space $(\Omega, \Sigma, P)$. We say that $\phi$ is stochastically integrable if it is weakly $L^{2}$ and there exists a random variable $\Phi \in L^{1}(\Omega, X)$ such that for all $x^{\prime} \in X^{\prime}$ we have $P$-almost surely

$$
\left\langle\Phi, x^{\prime}\right\rangle=\int_{M}\left\langle\phi(t), x^{\prime}\right\rangle \mathrm{d} W(t)
$$

where the right side is a scalar stochastic integral. In this situation we write

$$
\Phi=\int_{M} \phi(t) \mathrm{d} W(t)
$$


2.4 Remark. This definition differs slightly from [16, Def. 2.1], but is equivalent by [16, Theorem 2.3]. The stochastic integral of a function, when it exists, is a Gaussian random variable, and hence belongs to all $L^{p}(\Omega, X)$, $1 \leq p<\infty$; moreover, the different $L^{p}$ norms are comparable with constants depending only on the $p$ 's in question; not on the probability space $\Omega$ nor on the Banach space $X$.

The set of stochastically integrable functions $\phi: M \rightarrow X$ is a linear space, which is also preserved by point-wise application of $T \in \mathscr{L}(X)$, and the stochastic integrability of a function does not depend on the particular choice of the Gaussian random measure $W$.

A countably-valued function $\phi=\sum_{k=1}^{\infty} x_{k} 1_{E_{k}}$, where the $E_{k}$ are disjoint and of finite measure, is stochastically integrable if and only if the series $\sum_{k=1}^{\infty} x_{k} W\left(E_{k}\right)$ converges (say, in $L^{2}(\Omega, X)$, but this is equivalent to various other forms of convergence by results on Gaussian series), in which case its value is the integral $\int_{M} \phi \mathrm{d} W$.

We record a collection of useful results for checking stochastic integrability and estimating the result. The first two deal with situations were a function is appropriately dominated by a stochastically integrable one:

2.5 Theorem. ([16, 2.7]) Let each of $(M, \mu)$ and $(N, \nu)$ be $\sigma$-finite measure spaces. Let $\phi: M \rightarrow X$ and $\psi: N \rightarrow X$ be weakly $L^{2}$, let $\phi$ be stochastically integrable, and let

$$
\int_{N}\left\langle\psi(t), x^{\prime}\right\rangle^{2} \mathrm{~d} \nu(t) \leq \int_{M}\left\langle\phi(t), x^{\prime}\right\rangle^{2} \mathrm{~d} \mu(t)
$$

for all $x^{\prime} \in X^{\prime}$. Then also $\psi$ is stochastically integrable and for all $1 \leq p<\infty$

$$
\left(E\left|\int_{0}^{\infty} \psi(t) \mathrm{d} W_{\nu}(t)\right|_{X}^{p}\right)^{1 / p} \leq\left(E\left|\int_{0}^{\infty} \phi(t) \mathrm{d} W_{\mu}(t)\right|_{X}^{p}\right)^{1 / p}
$$

2.6 Proposition. ([12, 4.11]; [15, 4.5]) Let $(M, \mu)$ be a $\sigma$-finite measure space and $X$ a Banach space. Let $\phi: M \rightarrow X$ be stochastically integrable, and let $a: M \rightarrow \mathscr{L}(X)$ be strongly measurable with an $R$-bounded range. Then also $t \mapsto a(t) \phi(t)$ is stochastically integrable, and moreover

$$
E\left|\int_{M} a(t) \phi(t) \mathrm{d} W(t)\right|_{X} \leq \mathscr{R}(a) E\left|\int_{M} \phi(t) \mathrm{d} W(t)\right|_{X}
$$

The final result is concerned with the preservation of stochastic integrability in limit processes. 
2.7 Theorem. ([16, 6.6]) Let $X$ be a Banach space, which does not contain an isomorphic copy of $c_{0}$ as a closed subspace. Let $\phi_{n}:[0, \infty[\rightarrow X$ be strongly measurable stochastically integrable functions, and $\phi:[0, \infty[\rightarrow X$ a strongly measurable, weakly $L^{2}$ function. If

$$
\begin{aligned}
& \int_{0}^{\infty}\left\langle\phi_{n}(t)-\phi(t), x^{\prime}\right\rangle^{2} \mathrm{~d} t \rightarrow 0, \\
& \int_{0}^{\infty}\left\langle\phi_{n}(t), x^{\prime}\right\rangle^{2} \mathrm{~d} t \uparrow \int_{0}^{\infty}\left\langle\phi(t), x^{\prime}\right\rangle^{2} \mathrm{~d} t
\end{aligned}
$$

for all $x^{\prime} \in X^{\prime}$ as $n \rightarrow \infty$, and moreover

$$
\sup _{n \geq 1} E\left|\int_{0}^{\infty} \phi_{n}(t) \mathrm{d} B_{t}\right|_{X}<\infty
$$

then $\phi$ is stochastically integrable, and $\int_{0}^{\infty} \phi(t) \mathrm{d} B_{t}$ is the limit in $L^{p}(\Omega, X)$ of $\int_{0}^{\infty} \phi_{n}(t) \mathrm{d} B_{t}$ as $n \rightarrow \infty$.

This is formulated in [16] for separable Banach spaces, but the strong measurability of $\phi_{n}$ and $\phi$ ensures that they take their values (a.e.) in a separable subspace $E$ of $X$, so we are back to this setting.

\section{Necessity of the UMD condition}

We start the proof of Theorem 1.6 from its converse assertion, which states that the UMD framework is the most general in which results of the kind outlined in the Introduction can be hoped for. It is well-known that in order to prove the UMD condition (1.5), it is sufficient to show this estimate for some sufficiently rich class of martingales. Here it is convenient to assume that the underlying measure space is $[0,1]^{n+1}$, and for $k=1, \ldots, n$

$$
\begin{aligned}
& d_{k}\left(\theta_{1}, \ldots, \theta_{n+1}\right)=\phi_{k}\left(\theta_{1}, \ldots, \theta_{k}\right) \psi_{k}\left(\theta_{k+1}\right), \\
& \phi_{k} \in L^{p}\left([0,1]^{k}, X\right), \quad \psi_{k} \in L^{p}(0,1), \quad \int_{0}^{1} \psi_{k} \mathrm{~d} t=0 .
\end{aligned}
$$

Note that one can easily represent all finite Paley-Walsh martingales in this form, so it sufficient to prove (1.5) for this class.

By a simple approximation argument, we may further assume that all $\phi_{k}$ and $\psi_{k}$ are trigonometric polynomials, say

$$
\phi_{k}\left(\bar{\theta}_{k}\right)=\sum_{|\bar{j}| \leq L_{k}} a_{\bar{j}}^{(k)} e^{\mathbf{i} 2 \pi \bar{j} \cdot \bar{\theta}_{k}}, \quad \psi_{k}(\theta)=\sum_{0<|j| \leq K_{k}} b_{j}^{(k)} e^{\mathbf{i} 2 \pi j \theta}
$$

where we have employed the notations

$$
\bar{\theta}_{k}:=\left(\theta_{1}, \ldots, \theta_{k}\right) \quad \text { and } \quad|\bar{j}|:=\left|j_{1}\right|+\cdots+\left|j_{k}\right| \text {. }
$$


Let $N_{1}<N_{2}<\cdots$ be some natural numbers (to be chosen below), and denote also $\bar{N}_{k}:=\left(N_{1}, \ldots, N_{k}\right)$. Then, for a fixed $\bar{\theta}_{n+1} \in[0,1]^{n+1}$, we define

$$
\begin{aligned}
f_{k}(\theta) & :=\phi_{k}\left(\bar{\theta}_{k}+\bar{N}_{k} \theta\right) \varphi_{k}\left(\theta_{k+1}+N_{k+1} \theta\right) \\
& =\sum_{|\bar{j}| \leq L_{k}} \sum_{0<|j| \leq K_{k}} a_{\bar{j}}^{(k)} b_{j}^{(k)} e^{\mathbf{i} 2 \pi\left(\bar{j} \cdot \bar{\theta}_{k}+j \theta_{k+1}\right)} e^{\mathbf{i} 2 \pi\left(\bar{j} \cdot \bar{N}_{k}+j N_{k+1}\right) \theta} \\
& =: \sum_{A_{k} \leq j \leq B_{k}}\left(c_{j}^{(k)} e^{\mathbf{i} 2 \pi j \theta}+c_{-j}^{(k)} e^{-\mathbf{i} 2 \pi j \theta}\right)=: \sum_{A_{k} \leq j \leq B_{k}} \chi_{j}^{(k)}(\theta) .
\end{aligned}
$$

where $A_{k}:=N_{k+1}-L_{k} N_{k}>0$ and $B_{k}:=K_{k} N_{k+1}+L_{k} N_{k}$. This is J. Bourgain's transformation from [3], which he originally used to prove the necessity of UMD for the $L^{p}(0,1 ; X)$ boundedness of the Hilbert transform. It is also central in S. Guerre-Delebrière's proof [11], which we modify here for the present purpose, elaborating on the remark in [11] that its main theorem "is also true for some other operators [...] with 'nice' associated multiplier".

We denote by $\|f\|_{A}$ the sum of the norms of all Fourier coefficient of $f$, and note for later use that $\left\|f_{k}\right\|_{A} \leq\left\|\phi_{k}\right\|_{A}\left\|\psi_{k}\right\|_{A}$, no matter how $\bar{\theta}_{n+1}$ and $N_{1}<N_{2}<\cdots$ are chosen.

By assumption, we know that

$$
\begin{aligned}
c\|f-\hat{f}(0)\|_{L^{p}(0,1 ; X)} & \leq E\left\|\int_{0}^{\infty} t \frac{\partial}{\partial t} e^{-t(-\triangle)^{1 / 2}} f \frac{\mathrm{d} B_{t}}{t^{1 / 2}}\right\|_{L^{p}(0,1 ; X)} \\
& \leq C\|f\|_{L^{p}(0,1 ; X)}
\end{aligned}
$$

for all $f \in L^{p}(0,1 ; X)$. The idea of the proof is to apply this to $f=\sum_{k=1}^{n} f_{k}$ and to show that the stochastic integrals

$$
\int_{0}^{\infty} t \frac{\partial}{\partial t} e^{-t(-\triangle)^{1 / 2}} f_{k} \frac{\mathrm{d} B_{t}}{t^{1 / 2}}, \quad k=1, \ldots, n
$$

behave almost like independent random variables, provided we choose the sequence $N_{1}<N_{2}<\cdots$ to be sufficiently rapidly increasing.

We compute

$$
\int_{0}^{\infty} t \frac{\partial}{\partial t} P^{t} f_{k} \frac{\mathrm{d} B_{t}}{t^{1 / 2}}=\sum_{A_{k} \leq j \leq B_{k}} \int_{0}^{\infty}-t j e^{-t j} \frac{\mathrm{d} B_{t}}{t^{1 / 2}} \chi_{j}^{(k)}=: \sum_{A_{k} \leq j \leq B_{k}} \gamma_{j} \chi_{j}^{(k)} .
$$

The $\gamma_{j}$, as Brownian integrals, have jointly Gaussian distribution, and

$$
E\left(\gamma_{i} \gamma_{j}\right)=\int_{0}^{\infty} t^{2}|i||j| e^{-t(|i|+|j|)} \frac{\mathrm{d} t}{t}=\frac{|i||j|}{(|i|+|j|)^{2}} \leq \min \left(\frac{|i|}{|j|}, \frac{|j|}{|i|}\right) .
$$


Let $j \geq A_{k}$, and let us compute the distance of $\gamma_{j}$ from

$$
\Gamma_{k-1}:=\operatorname{span}\left(\gamma_{m}: m \leq B_{k-1}\right)
$$

in $L^{2}(\Omega)$. We first observe that the sequence $\left(\gamma_{m}\right)_{m=1}^{\infty}$ is linearly independent. In fact, if $\sum_{m=1}^{M} a_{m} \gamma_{m}=0$, then by the Itô isometry $\sum_{m=1}^{M} a_{m} t m e^{-t m} \equiv 0$ for $t \in] 0, \infty\left[\right.$, which after dividing by $t$ and denoting $r:=e^{-t}$ says that $\sum_{m=1}^{M} m a_{m} r^{m} \equiv 0$ for $\left.r \in\right] 0,1[$. The distance of interest is found by minimizing

$$
\left\|\gamma_{j}-\sum_{m \leq B_{k-1}} a_{m} \gamma_{m}\right\|_{L^{2}(\Omega)}^{2}=\frac{1}{4}-2 \sum_{m \leq B_{k-1}} a_{m} \frac{j m}{(j+m)^{2}}+\sum_{m, n \leq B_{k-1}} a_{m} a_{n} \frac{m n}{(m+n)^{2}} .
$$

Requiring the gradient to vanish we obtain the condition

$$
\sum_{n \leq B_{k-1}} a_{m} \frac{m n}{(m+n)^{2}}=\frac{j m}{(j+m)^{2}} \quad \text { for } \quad m \leq B_{k-1} .
$$

The mentioned linear independence implies that this $B_{k-1} \times B_{k-1}$ system is invertible. Observe that the matrix here only depends on

$$
B_{k-1}=K_{k-1} N_{k}+L_{k-1} N_{k-1},
$$

whereas the right side of the equation is

$$
\frac{j m}{(j+m)^{2}} \leq \frac{m}{j} \leq \frac{B_{k-1}}{A_{k}}
$$

and $A_{k}=N_{k+1}-L_{k} N_{k}$. Assuming that we have chosen $N_{1}<\ldots<N_{k}$, we can now take the next $N_{k+1}$ so large that the function $\gamma \in \Gamma_{k-1}$, which minimizes the distance $\left\|\gamma_{j}-\gamma\right\|_{L^{2}(\Omega)}$, is smaller in size than any preassigned number $\delta>0$. Denoting by $\Pi_{k-1}$ the orthogonal projection onto $\Gamma_{k-1}$, we have shown that $\left\|\Pi_{k-1} \gamma_{j}\right\|_{L^{2}(\Omega)}<\delta$ for all $k=2, \ldots, n$ and all $j \geq A_{k}$, provided the sequence $N_{1}<N_{2}<\cdots$ increases sufficiently rapidly. It follows that

$$
\begin{aligned}
& \left|E\left\|\sum_{k=1}^{n} \sum_{A_{k} \leq j \leq B_{k}}\left(\gamma_{j}-\Pi_{k-1} \gamma_{j}\right) \chi_{j}^{(k)}\right\|_{L^{p}(0,1 ; X)}-E\left\|\sum_{k=1}^{n} \sum_{A_{k} \leq j \leq B_{k}} \gamma_{j} \chi_{j}^{(k)}\right\|_{L^{p}(0,1 ; X)}\right| \\
& \leq \sum_{k=1}^{n} \sum_{A_{k} \leq j \leq B_{k}} E\left|\Pi_{k-1} \gamma_{j}\right|\left\|\chi_{j}^{(k)}\right\|_{L^{p}(0,1 ; X)} \\
& \leq \delta \sum_{k=1}^{n} \sum_{A_{k} \leq|j| \leq B_{k}}\left|c_{j}^{(k)}\right|_{X} \leq \delta \sum_{k=1}^{n}\left\|\phi_{k}\right\|_{A}\left\|\psi_{k}\right\|_{A} .
\end{aligned}
$$

If $i \in\left[A_{k}, B_{k}\right]$ and $j \in\left[A_{\ell}, B_{\ell}\right]$ for $k<\ell$, then $\gamma_{i}-\Pi_{k-1} \gamma_{i} \in \Gamma_{k} \subset \Gamma_{\ell-1}$ and $\gamma_{j}-\Pi_{\ell-1} \gamma_{j} \perp \Gamma_{\ell-1}$, so that $\gamma_{i}-\Pi_{k-1} \gamma_{i}$ and $\gamma_{j}-\Pi_{\ell-1} \gamma_{j}$ are orthogonal and therefore, being jointly Gaussian, also independent. 
Let $\left(B_{t}^{(k)}\right)_{t>0}$ for $k=1, \ldots, n$ be independent Brownian motions, and $\gamma_{j}^{(k)}$ and $\Pi_{\ell}^{(k)}$ be defined like $\gamma_{j}$ and $\Pi_{\ell}$ but using $B_{t}^{(k)}$ in place of $B_{t}$. Since the terms with different $k$ are independent in any case, it is clear that

$$
\begin{aligned}
& E\left\|\sum_{k=1}^{n} \sum_{A_{k} \leq j \leq B_{k}}\left(\gamma_{j}-\Pi_{k-1} \gamma_{j}\right) \chi_{j}^{(k)}\right\|_{L^{p}(0,1 ; X)} \\
&=E\left\|\sum_{k=1}^{n} \sum_{A_{k} \leq j \leq B_{k}}\left(\gamma_{j}^{(k)}-\Pi_{k-1}^{(k)} \gamma_{j}^{(k)}\right) \chi_{j}^{(k)}\right\|_{L^{p}(0,1 ; X)}
\end{aligned}
$$

On the other hand, one can repeat the estimate (3.2) also with the random variables $\gamma_{j}^{(k)}$ and projections $\Pi_{\ell}^{(k)}$. Combining these two estimates, and recalling notation, we obtain

$$
\begin{gathered}
\mid E\left\|\sum_{k=1}^{n} \int_{0}^{\infty} t \frac{\partial}{\partial t} e^{-t(-\triangle)^{1 / 2}} f_{k} \frac{\mathrm{d} B_{t}}{t^{1 / 2}}\right\|_{L^{p}(0,1 ; X)} \\
\quad-E\left\|\sum_{k=1}^{n} \int_{0}^{\infty} t \frac{\partial}{\partial t} e^{-t(-\triangle)^{1 / 2}} f_{k} \frac{\mathrm{d} B_{t}^{(k)}}{t^{1 / 2}}\right\|_{L^{p}(0,1 ; X)} \mid \\
\leq 2 \delta \sum_{k=1}^{n}\left\|\phi_{k}\right\|_{A}\left\|\psi_{k}\right\|_{A},
\end{gathered}
$$

and consequently

$$
\begin{aligned}
c\left\|\sum_{k=1}^{n} f_{k}\right\|_{L^{p}(0,1 ; X)} & -2 \delta \sum_{k=1}^{n}\left\|\phi_{k}\right\|_{A}\left\|\psi_{k}\right\|_{A} \\
& \leq E\left\|\sum_{k=1}^{n} \int_{0}^{\infty} t \frac{\partial}{\partial t} e^{-t(-\triangle)^{1 / 2}} f_{k} \frac{\mathrm{d} B_{t}^{(k)}}{t^{1 / 2}}\right\|_{L^{p}(0,1 ; X)} \\
& \leq C\left\|\sum_{k=1}^{n} f_{k}\right\|_{L^{p}(0,1 ; X)}+2 \delta \sum_{k=1}^{n}\left\|\phi_{k}\right\|_{A}\left\|\psi_{k}\right\|_{A} .
\end{aligned}
$$

The expression in the middle is invariant under changing $f_{k}$ to $\epsilon_{k} f_{k}$, where $\left(\epsilon_{k}\right)_{k=1}^{n} \in\{-1,+1\}^{n}$, and thus we obtain

$$
\begin{aligned}
c\left\|\sum_{k=1}^{n} \epsilon_{k} f_{k}\right\|_{L^{p}(0,1 ; X)}-2 \delta \sum_{k=1}^{n} & \left\|\phi_{k}\right\|_{A}\left\|\psi_{k}\right\|_{A} \\
& \leq C\left\|\sum_{k=1}^{n} f_{k}\right\|_{L^{p}(0,1 ; X)}+2 \delta \sum_{k=1}^{n}\left\|\phi_{k}\right\|_{A}\left\|\psi_{k}\right\|_{A} .
\end{aligned}
$$


Reorganizing, raising to the power of $p$ and using

$$
\left.(a+b)^{p} \leq(1-\eta)^{1-p} a^{p}+\eta^{1-p} b^{p} \quad \text { for } \quad \eta \in\right] 0,1[,
$$

and finally integrating with respect to $\bar{\theta}_{n+1}$ over $[0,1]^{n+1}$, we get

$$
\begin{gathered}
c^{p} \int_{0}^{1} \int_{[0,1]^{n+1}}\left|\sum_{k=1}^{n} \epsilon_{k} \phi_{k}\left(\bar{\theta}_{k}+\bar{N}_{k} \theta\right) \psi_{k}\left(\theta_{k+1}+N_{k+1} \theta\right)\right|_{X}^{p} \mathrm{~d} \bar{\theta}_{n+1} \mathrm{~d} \theta \\
\leq(1-\eta)^{1-p} C^{p} \iint\left|\sum_{k=1}^{n} \phi_{k}\left(\bar{\theta}_{k}+\bar{N}_{k} \theta\right) \psi_{k}\left(\theta_{k+1}+N_{k+1} \theta\right)\right|_{X}^{p} \mathrm{~d} \bar{\theta}_{n+1} \mathrm{~d} \theta \\
+\eta^{1-p}(4 \delta)^{p}\left(\sum_{k=1}^{n}\left\|\phi_{k}\right\|_{A}\left\|\psi_{k}\right\|_{A}\right)^{p} .
\end{gathered}
$$

Making a change of variable in the $\bar{\theta}_{n+1}$ integrations, the dependence of the integrals on $\theta$ disappears, and we are left with

$$
\begin{aligned}
\left\|\sum_{k=1}^{n} \epsilon_{k} d_{k}\right\|_{L^{p}\left([0,1]^{n+1}, X\right)}^{p} \leq(1 & -\eta)^{1-p} \frac{C^{p}}{c^{p}}\left\|\sum_{k=1}^{n} d_{k}\right\|_{L^{p}\left([0,1]^{n+1}, X\right)}^{p} \\
& +\eta^{1-p} \frac{(4 \delta)^{p}}{c^{p}}\left(\sum_{k=1}^{n}\left\|\phi_{k}\right\|_{A}\left\|\psi_{k}\right\|_{A}\right)^{p} .
\end{aligned}
$$

It remains to take the limits $\delta \downarrow 0$ and $\eta \downarrow 0$, in this order. Denoting by $\mathscr{U}_{p}(X)$ the smallest constant in the UMD condition (1.5), we have shown that $\mathscr{U}_{p}(X) \leq C / c$, where $c$ and $C$ are the constants from the square-function estimate (3.1).

\section{Martingale inequalities}

Having settled the necessity of the UMD condition for the theory we want to develop, we now investigate inequalities which we can derive from this condition. Our first result is purely martingale-theoretic; it is the vectorvalued extension of Stein's [21, Eq. (**), p. 115].

4.1 Proposition. Let $X$ be a UMD space, $1<p<\infty$, and $\left(\mathscr{F}_{j}\right)_{j=0}^{\infty}$ an increasing or decreasing filtration of a measure space $(M, \mathscr{M}, \mu)$. Let $E_{k}$ be the conditional expectation with respect to $\mathscr{F}_{k}, D_{k}:=E_{k}-E_{k-1}$, with $E_{-1}:=0$, and

$$
\sigma_{n}:=\frac{1}{n+1} \sum_{k=0}^{n} E_{k}=\sum_{k=0}^{n}\left(1-\frac{k}{n+1}\right) D_{k}
$$


be the related Césaro sums. Then there exists a constant $C=C_{p}(X)<\infty$ such that

$$
E\left\|\sum_{n=1}^{\infty} \gamma_{n} n^{1 / 2}\left(\sigma_{n}-\sigma_{n-1}\right) f\right\|_{p} \leq C\|f\|_{p},
$$

where the $\gamma_{j}$ are independent standard Gaussian variables.

In proving this result, we make use of another inequality of Stein, [21, Theorem 8, p. 103], which was already extended to UMD spaces by J. Bourgain [4]. A proof, omitted in [4], is found e.g. in [5].

4.2 Proposition ([4, 21]). Let $\mathscr{E}=\left(E_{j}\right)_{j=0}^{\infty}$ be a monotone sequence of conditional expectations on $(M, \mu)$. Then $\mathscr{E}$ is $R$-bounded on $L^{p}(\mu, X)$ for $p \in] 1, \infty[$, and in fact

$$
E\left\|\sum_{j=1}^{n} \varepsilon_{j} E_{j} f_{j}\right\|_{L^{p}(\mu, X)} \leq \mathscr{U} E\left\|\sum_{j=1}^{n} \varepsilon_{j} f_{j}\right\|_{L^{p}(\mu, X)},
$$

where $\mathscr{U}$ is the unconditionality constant of martingale differences in $L^{p}(\mu, X)$.

We actually use this inequality with standard Gaussian variables $\gamma_{j}$ instead of the $\varepsilon_{j}$. Such an estimate follows from the stated one by a simple randomization argument.

Proof of Proposition 4.1. We express the differences of interest as

$$
\begin{aligned}
\sigma_{n}-\sigma_{n-1} & =\frac{1}{n(n+1)} \sum_{k=1}^{n} k D_{k} \\
& =\frac{1}{n(n+1)}\left[\sum_{j=0}^{\left\lfloor\log _{2} n\right\rfloor} \Delta_{j} \sum_{2^{j-1}<k \leq 2^{j}} k D_{k}+\Delta_{\left\lfloor\log _{2} n\right\rfloor+1} \sum_{2^{\left\lfloor\log _{2} n\right\rfloor<k \leq n}} k D_{k}\right],
\end{aligned}
$$

where $\Delta_{j}:=E_{2^{j}}-E_{2^{j-1}}\left(\right.$ and $\left.E_{1 / 2}:=E_{0}\right)$. By using the partial summation formula

$$
\sum_{a<k \leq b} k D_{k}=\sum_{a<k \leq b}\left[\left(k E_{k}-(k-1) E_{k-1}\right)-E_{k-1}\right]=b E_{b}-a E_{a}-\sum_{a \leq k<b} E_{k}
$$

and the fact that $\Delta_{j} E_{2^{j-1}}=0$, we obtain

$$
\begin{aligned}
\sum_{n \geq 1} \gamma_{n} n^{1 / 2}\left(\sigma_{n}-\sigma_{n-1}\right) f & \\
= & \sum_{j \geq 0} \sum_{n \geq 2^{j}} \frac{n^{1 / 2} \gamma_{n}}{n(n+1)}\left(2^{j} E_{2^{j}}-\sum_{2^{j-1}<k<2^{j}} E_{k}\right) \Delta_{j} f \\
& +\sum_{j \geq 0} \sum_{2^{j} \leq n<2^{j+1}} \frac{n^{1 / 2} \gamma_{n}}{n(n+1)}\left(n E_{n}-\sum_{2^{j}<k<n} E_{k}\right) \Delta_{j+1} f=: I+I I
\end{aligned}
$$


So as to avoid problems of convergence, we can think that $\gamma_{n}$ denotes a Gaussian variable only for $1 \leq n<2^{N}$, say, whereas $\gamma_{n}:=0$ for $n \geq 2^{N}$. In the end we take the limit $N \rightarrow \infty$.

In the summation defining $I$ we further reorganize

$$
\sum_{n \geq 2^{j}}=\sum_{i \geq 0} \sum_{2^{j+i} \leq n<2^{j+i+1}}
$$

so as to arrive at

$$
\begin{aligned}
I & =\sum_{i \geq 0} 2^{-i} \sum_{j \geq 0} 2^{j+i} \sum_{2^{j+i} \leq n<2^{j+i+1}} \frac{n^{1 / 2} \gamma_{n}}{n(n+1)}\left(E_{2^{j}}-2^{-j} \sum_{2^{j-1}<k<2^{j}} E_{k}\right) \Delta_{j} f \\
& =: \sum_{i \geq 0} 2^{-i} I_{i} .
\end{aligned}
$$

By Prop. 4.2 , the set $\left(E_{k}\right)_{k=0}^{\infty}$, and then also its convex hull, is $R$-bounded on $L^{p}(\mu, X)$. In particular the operators

$$
E_{2^{j}}-2^{-j} \sum_{2^{j-1}<k<2^{j}} E_{k} \quad \text { resp. } 2^{-j} n E_{n}-2^{-j} \sum_{2^{j}<k<n} E_{k},
$$

where $j \geq 0,2^{j} \leq n<2^{j+1}$, belong to sets $\mathscr{T}_{i} \subset \mathscr{L}\left(L^{p}(\mu, X)\right), i=1,2$, respectively, with $\mathscr{R}\left(\mathscr{T}_{i}\right) \leq(i+1) \mathscr{U}$. Thus

$$
\begin{aligned}
& E\left\|I_{i}\right\|_{p} \leq 2 \mathscr{U} \cdot E\left\|\sum_{j \geq 0} 2^{j+i} \sum_{2^{j+i} \leq n<2^{j+i+1}} \frac{n^{1 / 2} \gamma_{n}}{n(n+1)} \Delta_{j} f\right\|_{p}, \\
& E\|I I\|_{p} \leq 3 \mathscr{U} \cdot E\left\|\sum_{j \geq 0} 2^{j} \sum_{2^{j} \leq n<2^{j+1}} \frac{n^{1 / 2} \gamma_{n}}{n(n+1)} \Delta_{j+1} f\right\|_{p}
\end{aligned}
$$

Let us introduce the new random variables

$$
g_{j}:=2^{j} \sum_{2^{j} \leq n<2^{j+1}} \frac{n^{1 / 2} \gamma_{n}}{n(n+1)}, \quad j \geq 0, \quad g_{-1}:=0 .
$$

These form a sequence of independent centred Gaussians, with variances

$$
\sigma_{j}^{2}:=E g_{j}^{2}=4^{j} \sum_{2^{j} \leq n<2^{j+1}} \frac{1}{n(n+1)^{2}} \leq 2^{j} \int_{2^{j}}^{\infty} \frac{\mathrm{d} t}{t^{2}}=1 .
$$

With the help of these we can write

$$
\begin{aligned}
\sum_{j \geq 0} 2^{j+i} \sum_{2^{j+i} \leq n<2^{j+i+1}} \frac{n^{1 / 2} \gamma_{n}}{n(n+1)} \Delta_{j} f & =\sum_{j \geq 0} g_{j+i} \Delta_{j} f, \\
\sum_{j \geq 0} 2^{j} \sum_{2^{j} \leq n<2^{j+1}} \frac{n^{1 / 2} \gamma_{n}}{n(n+1)} & =\sum_{j \geq 0} g_{j-1} \Delta_{j} f,
\end{aligned}
$$


so our task is reduced to proving that

$$
E\left\|\sum_{j \geq 0} g_{j+i} \Delta_{j} f\right\|_{p} \leq C\|f\|_{p} \quad \text { for } i=-1,0,1,2, \ldots
$$

But this follows from

$$
E\left\|\sum_{j \geq 0} g_{j+i} \Delta_{j} f\right\|_{p} \leq E\left\|\sum_{j \geq 0} \sigma_{j+i}^{-1} g_{j+i} \Delta_{j} f\right\|_{p} \leq C^{\prime} E\left\|\sum_{j \geq 0} \varepsilon_{j} \Delta_{j} f\right\|_{p} \leq C\|f\|_{p},
$$

where the first estimate is the contraction principle for Gaussian variables; the second is the equivalence in norm of normalized Gaussian and Rademacher random sequences - a result valid when the underlying Banach space has finite cotype (see [8, Theorem 12.27]), which every UMD space does; and the third is a randomized version of the UMD inequality.

\section{Weak Littlewood-Paley estimate for symmetric diffu- sion semigroups}

The goal of this section is to prove the following theorem, which is our vector-valued extension of Stein's [21, Eq. (1), p. 115] and the analogue of Martínez, Torrea and Xu's [14, Theorem 2.3]:

5.1 Theorem. Let $X$ be a UMD space, $1<p<\infty$ and $T^{t}$ be a symmetric diffusion semigroup on $(M, \mathscr{M}, \mu)$. Then there is a constant $C=C_{p}(X)<\infty$ such that

$$
E\left\|\int_{0}^{\infty} t^{1 / 2} \frac{\partial}{\partial t}\left(\frac{1}{t} \int_{0}^{t} T^{s} f \mathrm{~d} s\right) \mathrm{d} B_{t}\right\|_{L^{p}(\mu, X)} \leq C\|f\|_{L^{p}(\mu, X)}
$$

for all $f \in L^{p}(\mu, X)$. The existence of the stochastic integral is part of the conclusion.

Here, just like in [21] and [14], this result is derived as a corollary of the martingale inequality of Prop. 4.1 and the following celebrated representation theorem of G.-C. Rota [18] (cf. also [10, 21]):

5.2 Theorem ([18]). Let $Q \in \bigcup_{p \in[1, \infty]} L^{p}(\mu)$ be a bi-stochastic operator on $(M, \mathscr{M}, \mu)$. Then there exist the following objects: another measure space $(S, \mathscr{S}, \nu)$, a decreasing filtration

$$
\mathscr{S}=\mathscr{F}_{0} \supset \mathscr{F}_{1} \supset \mathscr{F}_{2} \supset \ldots,
$$


yet another $\sigma$-algebra $\hat{\mathscr{F}} \subset \mathscr{S}$, and a linear isomorphism

$$
J: \bigcup_{1 \leq p \leq \infty} L^{p}(M, \mathscr{M}, \mu) \rightarrow \bigcup_{1 \leq p \leq \infty} L^{p}(S, \hat{\mathscr{F}}, \nu)
$$

which preserves joint distributions, so in particular $L^{p}$ norms. Moreover, denoting by $\hat{E}$ and $E_{n}$ the conditional expectations with respect to $\hat{\mathscr{F}}_{\text {and }} \mathscr{F}_{n}$, we have

$$
Q^{2 n}=J^{-1} \hat{E} E_{n} J, \quad n=0,1,2, \ldots
$$

Since all the operators appearing in Rota's theorem are linear and positive, they have canonical tensor extensions of same norm to the Bochner spaces $L^{p}(\mu, X)$ for an arbitrary Banach space $X$. Thus we may apply Theorem 5.2 to the bi-stochastic operator $T^{\delta / 2}, \delta>0$, in our vector-valued setting.

Comparing the statements of Theorem 5.1 and Prop. 4.1, and making the heuristic approximations

$$
\begin{aligned}
\int_{0}^{\infty} F(t) \mathrm{d} B_{t} & \sim \sum_{n=1}^{\infty} F(\delta n)\left(B_{n \delta}-B_{(n-1) \delta}\right)=\sum_{n=1}^{\infty} F(\delta n) \delta^{1 / 2} \gamma_{n}, \\
\frac{\partial F(t)}{\partial t} & \sim \frac{F(n \delta)-F((n-1) \delta)}{\delta} \\
\frac{1}{t} \int_{0}^{t} T^{s} \mathrm{~d} s & \sim \frac{1}{n+1} \sum_{k=0}^{n} T^{\delta k}=J \hat{E}\left(\frac{1}{n+1} \sum_{k=0}^{n} E_{k}\right) J
\end{aligned}
$$

it seems quite plausible that Theorem 5.1 should follow from Prop. 4.1 as a limiting case when $\delta \downarrow 0$. Due to the rather subtle nature of the vector-valued stochastic integral, we provide this limiting argument in full detail.

Proof of Theorem 5.1. First of all, let us derive an immediate corollary of Prop. 4.1 and Rota's theorem applied to $Q:=T^{\delta / 2}$ for any $\delta>0$ :

$$
\begin{aligned}
& E\left\|\sum_{n=1}^{\infty} \gamma_{n} n^{1 / 2}\left(\frac{1}{n+1} \sum_{k=0}^{n} T^{\delta k} f-\frac{1}{n} \sum_{k=0}^{n-1} T^{\delta k} f\right)\right\|_{L^{p}(\mu, X)} \\
& =E\left\|\sum_{n=1}^{\infty} \gamma_{n} n^{1 / 2} J^{-1} \hat{E}\left(\frac{1}{n+1} \sum_{k=0}^{n} E_{k} J f-\frac{1}{n} \sum_{k=0}^{n-1} \hat{E} E_{k-1} J f\right)\right\|_{L^{p}(\mu, X)} \\
& =E\left\|\hat{E} \sum_{n=1}^{\infty} \gamma_{n} n^{1 / 2}\left(\sigma_{n}-\sigma_{n-1}\right) J f\right\|_{L^{p}(\nu, X)} \\
& \quad \leq E\left\|\sum_{n=1}^{\infty} \gamma_{n} n^{1 / 2}\left(\sigma_{n}-\sigma_{n-1}\right) J f\right\|_{L^{p}(\nu, X)} \leq C\|J f\|_{L^{p}(\nu, X)}=C\|f\|_{L^{p}(\mu, X)},
\end{aligned}
$$

where $C$ is the constant appearing in Prop. 4.1. 
Note that

$$
\frac{1}{n+1} \sum_{k=0}^{n} T^{\delta k}-\frac{1}{n} \sum_{k=0}^{n-1} T^{\delta k}=\frac{1}{n+1}\left(T^{\delta n}-\frac{1}{n} \sum_{k=0}^{n-1} T^{\delta k}\right),
$$

so applying the previous estimate to $T^{\delta u} f$ in place of $f$, we have

$$
\begin{aligned}
E\left\|\sum_{n=1}^{\infty} \gamma_{n} \frac{n^{1 / 2}}{n+1}\left(T^{\delta(n+u)} f-\frac{1}{n} \sum_{k=0}^{n-1} T^{\delta(k+u)} f\right)\right\|_{L^{p}(\mu, X)} \\
\leq C\left\|T^{\delta u} f\right\|_{L^{p}(\mu, X)} \leq C\|f\|_{L^{p}(\mu, X)} .
\end{aligned}
$$

Integration over $u \in[0,1]$ gives

$$
\begin{aligned}
E \| \sum_{n=1}^{\infty} & \gamma_{n} \frac{n^{1 / 2}}{n+1}\left(\int_{n}^{n+1} T^{\delta t} f \mathrm{~d} t-\frac{1}{n} \int_{0}^{n} T^{\delta t} f \mathrm{~d} t\right) \|_{L^{p}(\mu, X)} \\
& =E\left\|\sum_{n=1}^{\infty} \gamma_{n} \frac{n^{1 / 2}}{n+1} \int_{0}^{1}\left(T^{\delta(n+u)} f-\frac{1}{n} \sum_{k=0}^{n-1} T^{\delta(k+u)} f\right) \mathrm{d} u\right\|_{L^{p}(\mu, X)} \\
& \leq C\|f\|_{L^{p}(\mu, X)} .
\end{aligned}
$$

Now denote by $F(t)$ the $L^{p}(\mu, X)$-valued function whose stochastic integral we would like to estimate:

$$
F(t):=t^{1 / 2} \frac{\partial}{\partial t}\left(\frac{1}{t} \int_{0}^{t} T^{s} f \mathrm{~d} s\right)=t^{-1 / 2} T^{t} f-t^{-3 / 2} \int_{0}^{t} T^{s} f \mathrm{~d} s .
$$

It is seen at once, that the quantities estimated above are somewhat like the averages of $F(t)$ over intervals $[\delta n, \delta(n+1)]$. To make this more precise, for $n \geq 1$ we have

$$
\begin{aligned}
& \int_{n}^{n+1} F(\delta t) \mathrm{d} t \\
& =\delta^{-1 / 2} \int_{n}^{n+1} t^{-1 / 2} T^{\delta t} f \mathrm{~d} t-\delta^{-3 / 2} \int_{n}^{n+1} t^{-3 / 2}\left(\delta \int_{0}^{n} T^{\delta s} f \mathrm{~d} s+\delta \int_{n}^{t} T^{\delta s} \mathrm{~d} s\right) \mathrm{d} t \\
& =\delta^{-1 / 2}\left[\int_{n}^{n+1} T^{\delta t} f\left(t^{-1 / 2}-\int_{t}^{n+1} \frac{\mathrm{d} s}{s^{3 / 2}}\right) \mathrm{d} t-\int_{n}^{n+1} \frac{\mathrm{d} s}{s^{3 / 2}} \cdot \int_{0}^{n} T^{\delta t} f \mathrm{~d} t\right] \\
& =\delta^{-1 / 2}\left\{\int_{n}^{n+1} T^{\delta t} f \cdot \phi_{n}(t) \mathrm{d} t\right. \\
& \left.\quad+n \int_{n}^{n+1} \frac{\mathrm{d} s}{s^{3 / 2}}\left[\int_{n}^{n+1} T^{\delta t} f \mathrm{~d} t-\frac{1}{n} \int_{0}^{n} T^{\delta t} f \mathrm{~d} t\right]\right\},
\end{aligned}
$$


where, for $n \leq t \leq n+1$,

$$
\begin{aligned}
\phi_{n}(t) & :=t^{-1 / 2}-\int_{t}^{n+1} \frac{\mathrm{d} s}{s^{3 / 2}}-n \int_{n}^{n+1} \frac{\mathrm{d} s}{s^{3 / 2}} \\
& =-t^{-1 / 2}+\frac{2}{n^{1 / 2}+(n+1)^{1 / 2}}=-t^{-1 / 2}+u^{-1 / 2} \\
& =(t-u) \frac{2}{\xi^{3 / 2}} \in O\left(n^{-3 / 2}\right),
\end{aligned}
$$

where $n \leq u \leq n+1$ and $t \wedge u \leq \xi \leq t \vee u$, and the last two equalities, of course, we applications of mean-value theorems.

For $n=0$, one readily computes that

$$
\int_{0}^{1} F(\delta t) \mathrm{d} t=\delta^{-1 / 2} \int_{0}^{1} T^{\delta t} f \phi_{0}(t) \mathrm{d} t, \quad \phi_{0}(t)=2-t^{-1 / 2},
$$

and we have

$$
\int_{n}^{n+1}\left|\phi_{n}(t)\right| \mathrm{d} t \leq c(1+n)^{-3 / 2} \quad \text { for all } n=0,1,2, \ldots
$$

With $F_{\delta}:=\sum_{n=0}^{\infty} 1_{] \delta n, \delta(n+1)]} \cdot \int_{n}^{n+1} F(\delta s) \mathrm{d} s$, it follows that

$$
\begin{aligned}
& E\left\|\int_{0}^{\infty} F_{\delta}(t) \mathrm{d} B_{t}\right\|_{L^{p}(\mu, X)}=E\left\|\sum_{n \geq 0} \gamma_{n} \delta^{1 / 2} \int_{n}^{n+1} F(\delta t) \mathrm{d} t\right\|_{L^{p}(\mu, X)} \\
& \leq E\left\|\sum_{n \geq 0} \gamma_{n} \int_{n}^{n+1} T^{\delta t} f \cdot \phi_{n}(t) \mathrm{d} t\right\|_{L^{p}(\mu, X)} \\
& \quad+2 E\left\|\sum_{n \geq 1} \gamma_{n} \frac{n^{1 / 2}}{n+1}\left[\int_{n}^{n+1} T^{\delta t} f \mathrm{~d} t-\frac{1}{n} \int_{0}^{n} T^{\delta t} f \mathrm{~d} t\right]\right\|_{L^{p}(\mu, X)},
\end{aligned}
$$

where in the second term we applied the contraction principle and the fact that $n \int_{n}^{n+1} s^{-3 / 2} \mathrm{~d} s \leq 2 n^{1 / 2} /(n+1)$. For the second term we already know the upper estimate by $C\|f\|_{L^{p}(\mu, X)}$, whereas for the first one we have

$$
\begin{aligned}
& E\left\|\sum_{n \geq 0} \gamma_{n} \int_{n}^{n+1} T^{\delta t} f \phi_{n}(t) \mathrm{d} t\right\|_{L^{p}(\mu, X)} \\
& \quad \leq \sum_{n \geq 0} E\left|\gamma_{n}\right| \int_{n}^{n+1}\left\|T^{\delta t} f\right\|_{L^{p}(\mu, X)}\left|\phi_{n}(t)\right| \mathrm{d} t \leq C\|f\|_{L^{p}(\mu, X)}
\end{aligned}
$$

since $\left\|T^{\delta t} f\right\|_{p} \leq\|f\|_{p}$ and $\sum_{n=0}^{\infty} \int_{n}^{n+1}\left|\phi_{n}(t)\right| \mathrm{d} t \leq c \sum_{n=0}^{\infty}(1+n)^{-3 / 2}$ is summable. 
We have proved the stochastic integrability of $F_{\delta}$ with an estimate uniform in $\delta>0$. This implies in particular that the $F_{\delta}$ are weakly $L^{2}$, and that $\sup _{\delta>0}\left\|\left\langle F_{\delta}(\cdot), \lambda\right\rangle\right\|_{L^{2}(0, \infty)}<\infty$ for every $\lambda \in L^{p}(\mu, X)^{\prime}$. The functions $F_{2^{-k}}, k=1,2, \ldots$, form a martingale, and hence the membership of $F$ in weak $L^{2}$ follows by the maximal theorem:

$$
\begin{aligned}
\|\langle F(\cdot), \lambda\rangle\|_{L^{2}(0, \infty)} & \leq\left\|\sup _{k \in \mathbb{Z}_{+}}\left|\left\langle F_{2^{-k}}(\cdot), \lambda\right\rangle\right|\right\|_{L^{2}(0, \infty)} \\
& \leq 2 \sup _{k \in \mathbb{Z}_{+}}\left\|\left\langle F_{2^{-k}}(\cdot), \lambda\right\rangle\right\|_{L^{2}(0, \infty)}<\infty .
\end{aligned}
$$

The stochastic integrability of $F$ with the asserted estimate now follows from Theorem 2.7: The stochastic integrals of $F_{2^{-k}}$ are uniformly bounded, and by the martingale convergence theorem we have

$$
\begin{aligned}
& \int_{0}^{\infty}\left\langle F_{2^{-k}}(t)-F(t), \lambda\right\rangle^{2} \mathrm{~d} t \rightarrow 0, \\
& \int_{0}^{\infty}\left\langle F_{2^{-k}}(t), \lambda\right\rangle^{2} \mathrm{~d} t \uparrow \int_{0}^{\infty}\langle F(t), \lambda\rangle^{2} \mathrm{~d} t
\end{aligned}
$$

as $k \rightarrow \infty$ for all $\lambda \in L^{p}(\mu, X)^{\prime}$; moreover, the UMD space $L^{p}(\mu, X)$ cannot have $c_{0}$ as a subspace, so all the conditions of Theorem 2.7 are verified.

\section{Full Littlewood-Paley estimate for subordinated semi- groups}

Introducing the notation

$$
N_{t} f:=t \frac{\partial}{\partial t}\left(\frac{1}{t} \int_{0}^{t} T^{s} f \mathrm{~d} s\right)
$$

the main result of the previous section, Theorem 5.1, says that

$$
E\left\|\int_{0}^{\infty} N_{t} f \frac{\mathrm{d} B_{t}}{t^{1 / 2}}\right\|_{L^{p}(\mu, X)} \leq C\|f\|_{L^{p}(\mu, X)}
$$

where $C=C_{p}(X)<\infty$ when $X$ is a UMD space and $\left.p \in\right] 1, \infty[$.

When $P^{t}$ is the subordinated semigroup associated with $T^{t}$, the subordination formula

easily yields after a change of variable

$$
P^{t} f=\frac{1}{\sqrt{\pi}} \int_{0}^{\infty} \frac{e^{-u}}{\sqrt{u}} T^{t^{2} / 4 u} f \mathrm{~d} u
$$

$$
\begin{aligned}
t \frac{\partial}{\partial t} P^{t} f & =\int_{0}^{\infty} \varphi_{1}(u) N_{u t^{2}} f \mathrm{~d} u \\
\varphi_{1}(u) & :=-\frac{1}{\sqrt{\pi}} u \frac{\partial}{\partial u} \frac{e^{-1 / 4 u}}{u^{3 / 2}} \in L^{1}(0, \infty) .
\end{aligned}
$$


More generally, one can establish by repeated integration by parts that

$$
t^{k} \frac{\partial^{k}}{\partial t^{k}} P^{t} f=\int_{0}^{\infty} \varphi_{k}(u) N_{u t^{2}} f \mathrm{~d} u, \quad \varphi_{k} \in L^{1}(0, \infty), \quad k=1,2, \ldots
$$

We omit the details of the standard computations leading to these formulae.

A part of Theorem 1.6 is now an immediate corollary of Theorem 5.1:

Proof of the right side estimate in (1.7): By (6.1), it is enough to show that

$$
E\left\|\int_{0}^{\infty} t^{k} \frac{\partial^{k}}{\partial t^{k}} P^{t} f \frac{\mathrm{d} B_{t}}{t^{1 / 2}}\right\|_{L^{p}(\mu, X)} \leq C E\left\|\int_{0}^{\infty} N_{t} f \frac{\mathrm{d} B_{t}}{t^{1 / 2}}\right\|_{L^{p}(\mu, X)} .
$$

This follows from the following computation with an arbitrary $\lambda \in L^{p}(\mu, X)^{\prime}$ and Theorem 2.5:

$$
\begin{aligned}
\int_{0}^{\infty}\left\langle t^{k} \frac{\partial^{k}}{\partial t^{k}} P^{t} f, \lambda\right\rangle^{2} \frac{\mathrm{d} t}{t} & =\int_{0}^{\infty}\left\langle\int_{0}^{\infty} \varphi_{k}(u) N_{u t^{2}} f \mathrm{~d} u, \lambda\right\rangle^{2} \frac{\mathrm{d} t}{t} \\
& =\int_{0}^{\infty}\left(\int_{0}^{\infty} \varphi_{k}(u)\left\langle N_{u t^{2}} f, \lambda\right\rangle \mathrm{d} u\right)^{2} \frac{\mathrm{d} t}{t} \\
& \leq \int_{0}^{\infty}\left\|\varphi_{k}\right\|_{L^{1}(0, \infty)} \int_{0}^{\infty}\left|\varphi_{k}(u)\right|\left\langle N_{u t^{2}} f, \lambda\right\rangle^{2} \mathrm{~d} u \frac{\mathrm{d} t}{t} \\
& =\frac{1}{2}\left\|\varphi_{k}\right\|_{L^{1}(0, \infty)} \int_{0}^{\infty}\left|\varphi_{k}(u)\right| \int_{0}^{\infty}\left\langle N_{s} f, \lambda\right\rangle^{2} \frac{\mathrm{d} s}{s} \mathrm{~d} u \\
& =\frac{1}{2}\left\|\varphi_{k}\right\|_{L^{1}(0, \infty)}^{2} \int_{0}^{\infty}\left\langle N_{s} f, \lambda\right\rangle^{2} \frac{\mathrm{d} s}{s} .
\end{aligned}
$$

6.2 Remark. The above proved inequality becomes stronger with increasing $k$. In fact, independently of the underlying Banach space $X$, we have the estimate

$$
E\left\|\int_{0}^{\infty} t^{k} \frac{\partial^{k}}{\partial t^{k}} P^{t} f \frac{\mathrm{d} W_{t}}{t^{1 / 2}}\right\|_{L^{p}(\mu, X)} \leq \frac{1}{k} E\left\|\int_{0}^{\infty} t^{k+1} \frac{\partial^{k+1}}{\partial t^{k+1}} P^{t} f \frac{\mathrm{d} W_{t}}{t^{1 / 2}}\right\|_{L^{p}(\mu, X)},
$$

which contains the implicit statement that the existence of the stochastic integral on the right implies the existence of that on the left. 
This is again a consequence of Theorem 2.5:

$$
\begin{aligned}
\int_{0}^{\infty}\left\langle t^{k} \frac{\partial^{k}}{\partial t^{k}} P^{t} f, \lambda\right\rangle^{2} \frac{\mathrm{d} t}{t} & =\int_{0}^{\infty}\left(t^{k} \int_{t}^{\infty}\left\langle\frac{\partial^{k+1}}{\partial s^{k+1}} P^{s} f, \lambda\right\rangle s^{(k+1) / 2} \frac{\mathrm{d} s}{s^{(k+1) / 2}}\right)^{2} \frac{\mathrm{d} t}{t} \\
& \leq \int_{0}^{\infty} t^{2 k} \int_{t}^{\infty} s^{k+1}\left\langle\frac{\partial^{k+1}}{\partial s^{k+1}} P^{s} f, \lambda\right\rangle^{2} \mathrm{~d} s \times \int_{t}^{\infty} \frac{\mathrm{d} s}{s^{k+1}} \frac{\mathrm{d} t}{t} \\
& =\frac{1}{k} \int_{0}^{\infty} s^{k+1}\left\langle\frac{\partial^{k+1}}{\partial s^{k+1}} P^{s} f, \lambda\right\rangle^{2} \int_{0}^{s} t^{k-1} \mathrm{~d} t \mathrm{~d} s \\
& =\frac{1}{k^{2}} \int_{0}^{\infty} s^{2(k+1)}\left\langle\frac{\partial^{k+1}}{\partial s^{k+1}} P^{s} f, \lambda\right\rangle^{2} \frac{\mathrm{d} s}{s}
\end{aligned}
$$

We are left with proving the left inequality in (1.7), where the $k=1$ case suffices by Remark 6.2. This is achieved by a duality argument, based on the identity

$$
\left\langle f-E_{0} f, g\right\rangle=4 E\left\langle\int_{0}^{\infty} t^{1 / 2} \frac{\partial}{\partial t} P^{t} f \mathrm{~d} B_{t}, \int_{0}^{\infty} t^{1 / 2} \frac{\partial}{\partial t} P^{t} g \mathrm{~d} B_{t}\right\rangle,
$$

which we first establish for real-valued $f, g \in L^{2}(\mu)$.

In this situation, we have the representation $P^{t} f=\int_{[0, \infty[} e^{-\lambda t} \mathrm{~d} E_{\lambda} f$ provided by Hilbert space spectral theory, and then

$$
\begin{aligned}
t^{1 / 2} \frac{\partial}{\partial t} P^{t} f & =-t^{1 / 2} \int_{] 0, \infty[} \lambda e^{-\lambda t} \mathrm{~d} E_{\lambda} f, \\
\left\|t^{1 / 2} \frac{\partial}{\partial t} P^{t} f\right\|_{L^{2}(\mu)}^{2} & =t \int_{] 0, \infty[} \lambda^{2} e^{-2 \lambda t}\left\langle\mathrm{~d} E_{\lambda} f, f\right\rangle .
\end{aligned}
$$

An easy computation with Fubini's theorem now shows that

$$
\int_{0}^{\infty}\left\|t^{1 / 2} \frac{\partial}{\partial t} P^{t} f\right\|_{L^{2}(\mu)}^{2} \mathrm{~d} t=\frac{1}{4}\left\|f-E_{0} f\right\|_{L^{2}(\mu)}^{2},
$$

where we note that $E_{0}=E_{\{0\}}$ is the spectral projection on $L^{2}(\mu)$. By polarizing the previous identity, we obtain

$$
\int_{0}^{\infty}\left\langle t^{1 / 2} \frac{\partial}{\partial t} P^{t} f, t^{1 / 2} \frac{\partial}{\partial t} P^{t} g\right\rangle \mathrm{d} t=\frac{1}{4}\left\langle f-E_{0} f, g-E_{0} g\right\rangle=\frac{1}{4}\left\langle f-E_{0} f, g\right\rangle,
$$

and (6.3) follows from the Itô isometry on $L^{2}(\mu)$.

Obviously this identity also holds if $f \in L^{2}(\mu)$ is replaced by $f=\phi(\cdot) x$ with $\phi \in L^{2}(\mu)$ and $x \in X$, and similarly $g \in L^{2}(\mu)$ by $g=\psi(\cdot) x^{\prime}$ with $\psi \in L^{2}(\mu)$ and $x^{\prime} \in X^{\prime}$. By bi-linearity, it holds for $f \in X \otimes L^{2}(\mu)$ and $g \in X^{\prime} \otimes L^{2}(\mu)$. 
Applying the inequalities of Hölder and Khinchin-Kahane, (6.3) gives

$$
\begin{aligned}
& \left|\left\langle f-E_{0} f, g\right\rangle\right| \\
& \quad \leq C E\left\|\int_{0}^{\infty} t^{1 / 2} \frac{\partial}{\partial t} P^{t} f \mathrm{~d} W_{t}\right\|_{L^{p}(\mu, X)} \cdot E\left\|\int_{0}^{\infty} t^{1 / 2} \frac{\partial}{\partial t} P^{t} g \mathrm{~d} W_{t}\right\|_{L^{p^{\prime}\left(\mu, X^{\prime}\right)}} .
\end{aligned}
$$

If $X$ is assumed to be UMD and $1<p<\infty$, then also $X^{\prime}$ is UMD and $1<p^{\prime}<\infty$; thus

$$
E\left\|\int_{0}^{\infty} t^{1 / 2} \frac{\partial}{\partial t} P^{t} g \mathrm{~d} W_{t}\right\|_{L^{p^{\prime}\left(\mu, X^{\prime}\right)}} \leq C\|g\|_{L^{p^{\prime}\left(\mu, X^{\prime}\right)}} .
$$

It follows that

$$
\left\|f-E_{0} f\right\|_{L^{p}(\mu, X)}=\sup \left|\left\langle f-E_{0} f, g\right\rangle\right| \leq C E\left\|\int_{0}^{\infty} t^{1 / 2} \frac{\partial}{\partial t} P^{t} f \mathrm{~d} W_{t}\right\|_{L^{p}(\mu, X)},
$$

where the supremum is taken over all $g \in X^{\prime} \otimes\left[L^{2}(\mu) \cap L^{p^{\prime}}(\mu)\right]$ with $\|g\|_{L^{p^{\prime}}\left(\mu, X^{\prime}\right)} \leq 1$.

Thus we have shown the left estimate in (1.7) for all $f \in X \otimes\left[L^{2}(\mu) \cap L^{p}(\mu)\right]$. To get it for all $f \in L^{p}(\mu, X)$, we use the density of the above mentioned subspace and the fact that both sides of the desired inequality are continuous in $f \in L^{p}(\mu, X)$ by the boundedness of $E_{0}$ and right-hand inequality in (1.7), which was already proved.

This completes the proof of Theorem 1.6.

\section{The operator-valued Marcinkiewicz-Stein multiplier theorem}

We now consider the application of the Littlewood-Paley inequalities to the question of boundedness of the "multiplier operators"

$$
T_{a} f:=-\int_{0}^{\infty} a(t) \frac{\partial}{\partial t} P^{t} f \mathrm{~d} t
$$

where $a(t)$ is an $\mathscr{L}\left(L^{p}(\mu, X)\right)$-valued strongly measurable function which satisfies the commutation condition $a(t) P^{s}=P^{s} a(t)$ for all $0 \leq s, t<\infty$.

The $R$-boundedness condition of Theorem 1.9 can be slightly relaxed as follows: Let $\mathscr{T} \subset \mathscr{L}\left(L^{p}(\mu, X)\right)$ be an absolutely convex $R$-bounded set, closed in the strong operator topology. Let $\|\cdot\|_{\mathscr{T}}$ denote the Minkowski functional of $\mathscr{T}$. We assume that $a$ takes its values in span $\mathscr{T}$, and satisfies

$$
\sup _{0<T<\infty} \frac{1}{T} \int_{0}^{T}\|a(t)\|_{\mathscr{T}}^{2} \mathrm{~d} t<\infty
$$

Note that under the assumption of Theorem 1.9, $\mathscr{T}$ can be taken to be the strong operator closure of the absolute convex hull of range $(a)$, and the above 
supremum is 1 . In general we claim (and prove below) that Theorem 1.9 remains valid under the above stated assumptions on the multiplier $a$.

The following lemma shows that the defining formula (7.1) is meaningful:

7.2 Lemma. There is a dense subspace $F$ of $L^{p}(\mu, X)$ such that the integral defining $T_{a} f$ converges absolutely for every $f \in F$ and every strongly measurable a $:\left[0, \infty\left[\rightarrow \mathscr{L}\left(L^{p}(\mu, X)\right)\right.\right.$ such that

$$
\sup _{0<T<\infty} T^{-1 / 2}\|a\|_{L^{2}\left(0, T ; \mathscr{L}\left(L^{p}(\mu, X)\right)\right)}<\infty .
$$

Proof. Let $-A=-A_{p}$ denote the generator of $P^{t}$ on $L^{p}(\mu)$. We claim that

$$
L^{p}(\mu)=E_{0} L^{p}(\mu) \oplus \overline{\mathcal{D}\left(A_{p}\right) \cap \operatorname{range}\left(A_{p}\right)},
$$

and that we may take $F:=X \otimes\left[E_{0} L^{p}(\mu)+\left(\mathcal{D}\left(A_{p}\right) \cap \operatorname{range}\left(A_{p}\right)\right)\right]$ as the desired dense subspace. To prove (7.3), note first that $P^{t} \phi \in \mathcal{D}\left(A_{p}^{2}\right)$ for $t>0$ and $\phi \in L^{p}(\mu)$, and $A_{p} P^{t} \phi=-\partial P^{t} \phi / \partial t$. Thus every $\phi \in L^{p}(\mu)$ satisfies

$$
\begin{aligned}
\phi & =E_{0} \phi+\left(\phi-E_{0} \phi\right)=E_{0} \phi+\lim _{n \rightarrow \infty}\left(P^{1 / n} \phi-P^{n} \phi\right) \\
& =E_{0} \phi+\lim _{n \rightarrow \infty} \int_{1 / n}^{n} A_{p} P^{t} \phi \mathrm{d} t \in E_{0} L^{p}(\mu)+\overline{\mathcal{D}\left(A_{p}\right) \cap \operatorname{range}\left(A_{p}\right)} .
\end{aligned}
$$

If $\phi=A \varrho \in \operatorname{range}(A)$, then $P^{t} \phi=A P^{t} \varrho=-\partial P^{t} \varrho / \partial t$. Thus

$$
P^{n} \varrho-P^{n+1} \varrho=\int_{n}^{n+1} P^{t} \phi \mathrm{d} t \rightarrow E_{0} \phi \quad \text { as } \quad n \rightarrow \infty,
$$

but also $P^{n} \varrho \rightarrow E_{0} \varrho$, so that $E_{0} \phi=E_{0} \varrho-E_{0} \varrho=0$, and the sum is direct.

Since $\partial P^{t} \psi / \partial t \equiv 0$ for $\psi \in E_{0} L^{p}(\mu)$, it suffices to check the absolute convergence for $f=x \otimes \phi$ with $\phi=A \varrho \in \mathcal{D}(A) \cap \operatorname{range}(A)$. Then

$$
\begin{aligned}
\left\|a(t) \frac{\partial}{\partial t} P^{t} f\right\|_{L^{p}(\mu, X)} & =\left\|a(t)\left(x \otimes A P^{t} \phi\right)\right\|_{L^{p}(\mu, X)} \\
& \leq\|a(t)\|_{\mathscr{L}\left(L^{p}(\mu, X)\right)}|x|_{X}\left\|A P^{t} \phi\right\|_{L^{p}(\mu)},
\end{aligned}
$$

For the last $L^{p}(\mu)$ norm we have the upper bounds $\left\|P^{t} A \phi\right\|_{p} \leq\|A \phi\|_{p}$ and $\left\|A^{2} P^{t} \varrho\right\|_{p} \leq C t^{-2}\|\varrho\|_{p}$.

Thus we have

$$
\begin{aligned}
& \int_{0}^{\infty}\left\|a(t) \frac{\partial}{\partial t} P^{t} f\right\|_{L^{p}(\mu, X)} \mathrm{d} t \\
& \leq C \int_{0}^{\infty}\|a(t)\|(1+t)^{-2} \mathrm{~d} t=2 C \int_{0}^{\infty}\|a(t)\| \int_{t}^{\infty}(1+s)^{-3} \mathrm{~d} s \mathrm{~d} t \\
& =2 C \int_{0}^{\infty}(1+s)^{-3} \int_{0}^{s}\|a(t)\| \mathrm{d} t \mathrm{~d} s \leq \tilde{C} \int_{0}^{\infty}(1+s)^{-3} s \mathrm{~d} s<\infty .
\end{aligned}
$$


Theorem 1.9 will be a consequence of Theorem 1.6 and the following:

7.4 Proposition. Let $a:\left[0, \infty\left[\rightarrow \mathscr{L}\left(L^{p}(\mu, X)\right)\right.\right.$ have the properties listed in the beginning of the section. Let $f \in F$ and let

$$
t \mapsto t^{3 / 2} \partial^{2} P^{t} f / \partial t
$$

be stochastically integrable on $\left[0, \infty\left[\right.\right.$. Then also $s \mapsto s^{1 / 2} \partial P^{s} T_{a} f / \partial s$ is stochastically integrable, and

$$
\begin{aligned}
& E\left\|\int_{0}^{\infty} s^{1 / 2} \frac{\partial}{\partial s} P^{s} T_{a} f \mathrm{~d} B_{s}\right\|_{L^{p}(\mu, X)} \\
& \quad \leq \mathscr{R}(\mathscr{T}) \sup _{0<T<\infty}\left(\frac{1}{T} \int_{0}^{T}\|a(t)\|_{\mathscr{T}}^{2} \mathrm{~d} t\right)^{\frac{1}{2}} E\left\|\int_{0}^{\infty} t^{3 / 2} \frac{\partial^{2}}{\partial t^{2}} P^{t} f \mathrm{~d} B_{t}\right\|_{L^{p}(\mu, X)} .
\end{aligned}
$$

Proof. First note that

$$
\frac{\partial}{\partial s} P^{s} T_{a} f=-\int_{0}^{\infty} a(t) \frac{\partial^{2}}{\partial t^{2}} P^{t+s} f \mathrm{~d} t=-\int_{s}^{\infty} \frac{1}{t} \cdot a(t-s) t \frac{\partial^{2}}{\partial t^{2}} P^{t} f \mathrm{~d} t .
$$

For every $\lambda \in L^{p}(\mu, X)^{\prime}$ we have

$$
\begin{aligned}
\int_{0}^{\infty}\left\langle s^{1 / 2} \frac{\partial}{\partial s} P^{s} F, \lambda\right\rangle^{2} \mathrm{~d} s & =\int_{0}^{\infty}\left(\int_{s}^{\infty} \frac{s^{1 / 2}}{t}\left\langle a(t-s) t \frac{\partial^{2}}{\partial t^{2}} P^{t} f, \lambda\right\rangle \mathrm{d} t\right)^{2} \mathrm{~d} s \\
& \leq \int_{0}^{\infty} \int_{s}^{\infty} \frac{s}{t^{2}} \mathrm{~d} t \cdot \int_{s}^{\infty}\left\langle a(t-s) t \frac{\partial^{2}}{\partial t^{2}} P^{t} f, \lambda\right\rangle^{2} \mathrm{~d} t \mathrm{~d} s \\
& =\int_{0}^{\infty} \int_{s}^{\infty}\left\langle a(t-s) t \frac{\partial^{2}}{\partial t^{2}} P^{t} f, \lambda\right\rangle^{2} \mathrm{~d} t \mathrm{~d} s .
\end{aligned}
$$

It follows from Theorem 2.5 that if

$$
(s, t) \mapsto 1_{\{t>s\}} a(t-s) t \partial^{2} P^{t} f / \partial t^{2}
$$

is integrable with respect to the Brownian sheet on $[0, \infty]^{2}$, then

$$
s \mapsto s^{1 / 2} \partial P^{s} F / \partial s
$$

is integrable with respect to the Brownian motion on $[0, \infty[$, and

$$
\begin{array}{rl}
E \| \int_{0}^{\infty} s^{1 / 2} \frac{\partial}{\partial s} P^{s} F & \mathrm{~d} B_{s} \|_{L^{p}(\mu, X)} \\
\leq E\left\|\iint_{\left[0, \infty\left[\left[^{2}\right.\right.\right.} 1_{\{t>s\}} a(t-s) t \frac{\partial^{2}}{\partial t^{2}} P^{t} f \mathrm{~d} W(s, t)\right\|_{L^{p}(\mu, X)} & .
\end{array}
$$


$\operatorname{But}(s, t) \mapsto 1_{\{t>s\}} a(t-s) /\|a(t-s)\|_{\mathscr{T}}($ understood as zero if $a(t-s)=0)$ is a strongly measurable function with values in the $R$-bounded set $\mathscr{T}$, and so, by Prop. 2.6, the function $1_{\{t>s\}} a(t-s) t \partial^{2} P^{t} f / \partial t^{2}$ is stochastically integrable if $1_{\{t>s\}}\|a(t-s)\|_{\mathscr{T}} t \partial^{2} P^{t} f / \partial t^{2}$ is, and in this case the inequality above can be continued with

$$
\leq \mathscr{R}(\mathscr{T}) E\left\|\iint_{\left[0, \infty\left[^{2}\right.\right.} 1_{\{t>s\}}\right\| a(t-s)\left\|_{\mathscr{T}} t \frac{\partial^{2}}{\partial t^{2}} P^{t} f \mathrm{~d} W(s, t)\right\|_{L^{p}(\mu, X)} .
$$

The existence of this last stochastic integral in turn follows by Theorem 2.5 from the estimate

$$
\begin{aligned}
\iint_{\left[0, \infty\left[^{2}\right.\right.} & \left\langle 1_{\{t>s\}}\|a(t-s)\|_{\mathscr{T}} t \frac{\partial^{2}}{\partial t^{2}} P^{t} f, \lambda\right\rangle^{2} \mathrm{~d} s \mathrm{~d} t \\
& =\int_{0}^{\infty} \frac{1}{t} \int_{0}^{t}\|a(t-s)\|_{\mathscr{T}}^{2} \mathrm{~d} s\left\langle t^{3 / 2} \frac{\partial^{2}}{\partial t^{2}} P^{t} f, \lambda\right\rangle^{2} \mathrm{~d} t \\
& \leq \sup _{0<t<\infty} \frac{1}{t} \int_{0}^{t}\|a(s)\|_{\mathscr{T}}^{2} \mathrm{~d} s \times \int_{0}^{\infty}\left\langle t^{3 / 2} \frac{\partial^{2}}{\partial t^{2}} P^{t} f, \lambda\right\rangle^{2} \mathrm{~d} t
\end{aligned}
$$

and the assumed stochastic integrability of $t^{3 / 2} \partial^{2} P^{t} f / \partial t^{2}$.

Proof of Theorem 1.9. Let $F$ be the dense subspace of $L^{p}(\mu, X)$ from Lemma 7.2, and let $f \in F$. Then

$$
E_{0} T_{a} f=-\int_{0}^{\infty} E_{0} a(t) \frac{\partial}{\partial t} P^{t} f \mathrm{~d} t=-\int_{0}^{\infty} a(t) E_{0} \frac{\partial}{\partial t} P^{t} f \mathrm{~d} t=0,
$$

since $E_{0}$, as the strong $\operatorname{limit} \lim _{s \rightarrow \infty} P^{s}$, commutes with $a(t)$, and

$$
E_{0} \frac{\partial}{\partial t} P^{t}=-E_{0} A_{p} P^{t}=0 \quad \text { on } \quad L^{p}(\mu) \quad \text { by }(7.3) .
$$

Thus by Lemma 7.2, Prop. 7.4, and Theorem 1.6,

$$
\begin{aligned}
\left\|T_{a} f\right\|_{L^{p}(\mu, X)} & =\lim _{n \rightarrow \infty}\left\|T_{a 1_{[1 / n, n]}} f\right\|_{L^{p}(\mu, X)} \\
& =\lim _{n \rightarrow \infty}\left\|\left(\mathrm{id}-E_{0}\right) T_{a 1_{[1 / n, n]}} f\right\|_{L^{p}(\mu, X)} \\
& \leq \liminf _{n \rightarrow \infty} \frac{1}{c} E\left\|\int_{0}^{\infty} s^{1 / 2} \frac{\partial}{\partial s} P^{s} T_{a 1_{[1 / n, n]}} f \mathrm{~d} B_{s}\right\|_{L^{p}(\mu, X)} \\
& \leq \frac{1}{c} \mathscr{R}(a) E\left\|\int_{0}^{\infty} t^{3 / 2} \frac{\partial^{2}}{\partial t^{2}} P^{t} f \mathrm{~d} B_{t}\right\|_{L^{p}(\mu, X)} \leq \frac{C}{c} \mathscr{R}(a)\|f\|_{L^{p}(\mu, X)},
\end{aligned}
$$

where $c$ and $C$ are constants from (1.7) for $k=1$ and $k=2$, respectively. This estimate allows the extension of $T_{a}$ to a bounded linear operator on all of $L^{p}(\mu, X)$. 
7.5 Remark. A simplification of the proof of Prop. 7.4 can be made for a scalar-valued multiplier $a \in L^{\infty}(0, \infty ; \mathbb{R})$. In this case, we may compute directly

$$
\begin{aligned}
\int_{0}^{\infty}\left\langle s^{1 / 2} \frac{\partial}{\partial s} P^{s} F, \lambda\right\rangle^{2} \mathrm{~d} s & \leq \int_{0}^{\infty} \int_{s}^{\infty}\left\langle a(t-s) t \frac{\partial^{2}}{\partial t^{2}} P^{t} f, \lambda\right\rangle^{2} \mathrm{~d} t \mathrm{~d} s \\
& =\int_{0}^{\infty} \frac{1}{t} \int_{0}^{t} a^{2}(t-s) \mathrm{d} s\left\langle t^{3 / 2} \frac{\partial^{2}}{\partial t^{2}} P^{t} f, \lambda\right\rangle^{2} \mathrm{~d} t \\
& \leq \sup _{0<t<\infty} \frac{1}{t} \int_{0}^{t}|a(s)|^{2} \mathrm{~d} s \times \int_{0}^{\infty}\left\langle t^{3 / 2} \frac{\partial^{2}}{\partial t^{2}} P^{t} f, \lambda\right\rangle^{2} \mathrm{~d} t
\end{aligned}
$$

and apply Theorem 2.5. Of course $a \in L^{\infty}(0, \infty ; \mathbb{C})$ can be handled similarly after first splitting into real and imaginary parts.

\section{Connection to holomorphic functional calculus}

It seems appropriate to comment on the relation of our Theorem 1.9 to the existence of an $H^{\infty}$ functional calculus for the negative generator $A$ of $P^{t}=e^{-t A}$; this is the framework in which results similar to ours have recently been proved by Kalton and Weis [12]. An excellent general reference to this notion is the already classic paper [7] of M. Cowling, I. Doust, A. McIntosh and A. Yagi. In a context similar to the present one, this problem was considered by Clément and Prüss [6], and following them we define

$$
\begin{aligned}
\tilde{a}(A) f & :=\int_{0}^{\infty} a(t) A e^{-t A} f \mathrm{~d} t=T_{a} f \quad \text { for } \\
\tilde{a}(\lambda) & :=\lambda \int_{0}^{\infty} a(t) e^{-t \lambda} \mathrm{d} t .
\end{aligned}
$$

By the results of the previous section, this gives rise to a bounded operator on $L^{p}(\mu, X)$ for every $a \in \mathscr{A}$, where

$$
\mathscr{A}:=\left\{a \in L_{\mathrm{loc}}^{2}(0, \infty ; \mathbb{C}): \sup _{0<T<\infty} T^{-1 / 2}\|a\|_{L^{2}(0, T)}<\infty\right\} .
$$

Let us denote by $\tilde{\mathscr{A}}$ the set of functions $\tilde{a}$ obtained from $a \in \mathscr{A}$ by the modified Laplace transformation in (8.1).

It is easy to see that every $\tilde{a} \in \tilde{\mathscr{A}}$ is a holomorphic function in the right half-plane $\mathbb{C}_{+} \equiv \Sigma_{\pi / 2}$, and moreover a bounded holomorphic function in every sector $\Sigma_{\theta}:=\{\lambda \in \mathbb{C} \backslash\{0\}:|\arg (\lambda)|<\theta\}$ with $\theta<\pi / 2$; we denote this last mentioned class by $H^{\infty}\left(\Sigma_{\theta}\right)$. On the other hand, we have

8.2 Lemma. $H^{\infty}\left(\mathbb{C}_{+}\right) \subset \tilde{\mathscr{A}}$, and this containment is strict. 
Proof. Let $h \in H^{\infty}\left(\mathbb{C}_{+}\right)$. Then in particular $\xi \mapsto h(\sigma+\mathbf{i} \xi) \in L^{\infty}(\mathbb{R})$, and hence $\xi \mapsto(\sigma+\mathbf{i} \xi)^{-1} h(\sigma+\mathbf{i} \xi) \in L^{2}(\mathbb{R})$ for $\sigma>0$. By the Fourier inversion theorem, there exists $b_{\sigma} \in L^{2}(\mathbb{R})$ such that, in the sense of $L^{2}$ convergence, we have

$$
b_{\sigma}(t)=\lim _{R \rightarrow \infty} \frac{1}{2 \pi} \int_{-R}^{R} \frac{h(\sigma+\mathbf{i} \xi)}{\sigma+\mathbf{i} \xi} e^{\mathbf{i} \xi t} \mathrm{~d} \xi=\lim _{R \rightarrow \infty} \frac{e^{-\sigma t}}{2 \pi \mathbf{i}} \int_{\sigma-\mathbf{i} R}^{\sigma+\mathbf{i} R} \frac{h(\lambda)}{\lambda} e^{\lambda t} \mathrm{~d} \lambda .
$$

For a subsequence $R_{n} \rightarrow \infty$, this limit exists for a.e. $t$, and by Cauchy's theorem the value of $\int_{\sigma-\mathbf{i} \infty}^{\sigma+\mathbf{i} \infty} h(\lambda) e^{\lambda t} \mathrm{~d} \lambda / \lambda$ is independent of $\sigma>0$. Thus there exists a measurable function $a$ on $\mathbb{R}$ such that for all $\sigma>0$ we have $b_{\sigma}(t)=e^{-\sigma t} a(t)$ for a.e. $t \in \mathbb{R}$. If $t<0$, we can close the integration path in (8.3) with a semicircle of radius $R$ in the right half-plane, and we find that $b_{\sigma}(t)=0=a(t)$ for all negative $t$. Thus

$$
\frac{h(\sigma+\mathbf{i} \xi)}{\sigma+\mathbf{i} \xi}=\lim _{R \rightarrow \infty} \int_{-R}^{R} b_{\sigma}(t) e^{-\mathbf{i} \xi t} \mathrm{~d} t=\lim _{R \rightarrow \infty} \int_{0}^{R} a(t) e^{-(\sigma+\mathbf{i} \xi) t} \mathrm{~d} t
$$

in the sense of $L^{2}$ convergence, and we need to check that $a$ has the desired estimate.

Since multiplication by the bounded function $1_{[0, T]}(t) e^{\sigma t}$ is a bounded operator on $L^{2}(\mathbb{R})$, the $L^{2}$ convergence (8.3) implies that

$$
\begin{aligned}
\frac{1}{T} & \int_{0}^{T}|a(t)|^{2} \mathrm{~d} t=\frac{1}{(2 \pi)^{2}} \lim _{R \rightarrow \infty} \frac{1}{T} \int_{0}^{T}\left|\int_{\sigma-\mathbf{i} R}^{\sigma+\mathbf{i} R} \frac{h(\lambda)}{\lambda} e^{\lambda t} \mathrm{~d} \lambda\right|^{2} \mathrm{~d} t \\
& =\frac{1}{(2 \pi)^{2}} \lim _{R \rightarrow \infty} \int_{-R}^{R} \int_{-R}^{R} \frac{h(\sigma+\mathbf{i} \xi) \bar{h}(\sigma+\mathbf{i} \eta)}{(\sigma+\mathbf{i} \xi)(\sigma+\mathbf{i} \eta)} \frac{e^{T(2 \sigma+\mathbf{i}(\xi-\eta))}-1}{T(2 \sigma+\mathbf{i}(\xi-\eta)} \mathrm{d} \xi \mathrm{d} \eta \\
& \leq \frac{\|h\|_{\infty}^{2}}{(2 \pi)^{2}} \frac{e^{2 \sigma T}+1}{T \sigma} \iint_{\mathbb{R}^{2}} \frac{1}{|1+\mathbf{i} \xi / \sigma| \cdot|1+\mathbf{i} \eta / \sigma| \cdot|2+\mathbf{i}(\xi-\eta) / \sigma|} \frac{\mathrm{d} \xi}{\sigma} \frac{\mathrm{d} \eta}{\sigma}
\end{aligned}
$$

The double integral is convergent, and after a change of variable, it is seen to be independent of $\sigma>0$. With $\sigma=T^{-1}$, we see that $T^{-1 / 2}\|a\|_{L^{2}(0, T)} \leq$ $C\|h\|_{\infty}$, which shows the asserted inclusion.

That $\tilde{A} \not \subset H^{\infty}\left(\mathbb{C}_{+}\right)$follows e.g. by considering the function

$$
a=\sum_{n=0}^{\infty} 1_{[2 n, 2 n+1]} \in L^{\infty}(0, \infty) .
$$

Its modified Laplace transform is $\tilde{a}(\lambda)=\left(1+e^{-\lambda}\right)^{-1}$, which has poles at $\lambda=\mathbf{i} \pi(2 n+1), n \in \mathbb{Z}$, and so is unbounded in $\mathbb{C}_{+}$.

Recalling that the generator of a subordinated semigroup $P^{t}$ is, by definition, $-A^{1 / 2}$, where $-A$ is the generator of a symmetric diffusion semigroup $T^{t}$, we can state the following result. 
8.4 Corollary. Let $T^{t}=e^{-t A}$ be a symmetric diffusion semigroup on some $(M, \mu)$. Let $X$ be a UMD space and $p \in] 1, \infty[$. Then $A$ has a bounded $H^{\infty}\left(\Sigma_{\pi}\right)$ functional calculus on $L^{p}(\mu, X)$.

Proof. It follows from Lemma 8.2 and the Marcinkiewicz-Stein multiplier theorem that $h\left(A^{1 / 2}\right)=h \circ \sqrt{\cdot}(A)$ defines a bounded operator on $L^{p}(\mu, X)$ for every $h \in H^{\infty}\left(\Sigma_{\pi / 2}\right)=H^{\infty}\left(\mathbb{C}_{+}\right)$. This is equivalent to saying that $g(A)$ defines a bounded operator on the same space for every $g \in H^{\infty}\left(\Sigma_{\pi}\right)=$ $\left.\left.H^{\infty}(\mathbb{C} \backslash]-\infty, 0\right]\right)$.

\section{Improved inequalities in certain interpolation spaces}

We have seen how the UMD property and the relation of semigroups and conditional expectations provided by Rota's theorem yield the weak LittlewoodPaley inequality of Theorem 5.1 for a general symmetric diffusion semigroup $T^{t}$.

It is discussed by Stein [21, §IV .5] why this is "the strongest relevant conclusion [for general diffusion semigroups] we can possibly hope to squeeze out of martingale theory", already in the scalar-valued case. Above we strengthened this conclusion by restricting to the subordinated semigroups.

We now present a different approach which applies to all symmetric diffusion semigroups, at the cost of reducing the admissible class of Banach spaces.

Here we consider the class of the (necessarily UMD) Banach spaces $X$, which are isomorphic to a closed subspace of a complex interpolation space $[H, Y]_{\theta}$, where $H$ is a Hilbert space, $Y$ is a UMD space, and $0<\theta<1$.

This class of spaces has appeared recently in the investigation of functional calculi for power-bounded operators by E. Berkson and T. A. Gillespie [2], and it contains all the standard examples of UMD spaces: every UMD-lattice of functions on a $\sigma$-finite measure space by a result of J.L. Rubio de Francia [19], the reflexive Sobolev spaces (which are subspaces of products of $L^{p}$ spaces), and the reflexive Schatten-von Neumann ideals $\mathscr{C}^{p}=\left[\mathscr{C}^{2}, \mathscr{C}^{q}\right]_{\theta}, 1 / p=(1-\theta) / 2+\theta / q$.

Actually, by results due to P. G. Dodds, T. K. Dodds and B. de Pagter [9], the interpolation properties of non-commutative spaces coincide with those of their commutative counterparts under fairly general conditions (see [9] for details), and so the theorem of Rubio de Francia implies the interpolation property for many more operator ideals. The interesting question posed in [19], whether the described interpolation class actually exhaust all UMD spaces, appears to remain open.

To see what can be done in these spaces in the present context, let us first note that the right-hand estimate in (1.7) holds true for every symmetric 
diffusion semigroup $T^{t}$ in place of $P^{t}$ if $X=H$ is a Hilbert space and $p=2$. We write this in the equivalent form

$$
E\left\|\int_{0}^{\infty} t \frac{\partial}{\partial t}\left(t^{k-1} \frac{\partial^{k-1}}{\partial t^{k-1}} T^{t}\right) f \frac{\mathrm{d} B_{t}}{t^{1 / 2}}\right\|_{L^{2}(\mu, H)} \leq c_{k}\|f\|_{L^{2}(\mu, H)}, \quad k=1,2, \ldots
$$

to make the connection with Theorem 5.1 more transparent. The proof of (9.1) is a routine computation with spectral integrals, observing that the stochastic $g$-function here reduces to the classical one by the Itô isometry.

In order to apply holomorphic interpolation, we employ the fractional integral defined for $\operatorname{Re} \alpha>0$ by

$$
I^{\alpha} \phi(t):=\frac{1}{\Gamma(\alpha)} \int_{0}^{t}(t-s)^{\alpha-1} \phi(s) \mathrm{d} s=\frac{t^{\alpha}}{\Gamma(\alpha)} \int_{0}^{1}(1-u)^{\alpha-1} \phi(t u) \mathrm{d} u,
$$

or more precisely its modification $M^{\alpha} \phi(t):=t^{-\alpha} I^{\alpha} \phi(t)$. We consider the action of these operators on functions $\phi$, which are $C^{\infty}$ on $] 0, \infty[$ and locally integrable on $\left[0, \infty\left[\right.\right.$. Recall the standard property $I^{\alpha+\beta}=I^{\alpha} I^{\beta}$ for $\operatorname{Re} \alpha, \operatorname{Re} \beta>0$. Clearly $I^{1}$ is just the usual integral $\int_{0}^{t}$.

By splitting the defining integral and integrating by parts, one finds that

$$
\begin{aligned}
M^{\alpha} \phi(t)= & \frac{1}{\Gamma(\alpha)} \int_{0}^{1 / 2}(1-u)^{\alpha-1} \phi(t u) \mathrm{d} u \\
& +\sum_{k=0}^{n-1} \frac{1}{2^{\alpha} \Gamma(\alpha+k+1)}\left(\frac{t}{2}\right)^{k} D^{k} \phi\left(\frac{t}{2}\right) \\
& +\frac{1}{\Gamma(\alpha+n)} \int_{1 / 2}^{1}(1-u)^{\alpha+n-1} t^{n} D^{n} \phi(t u) \mathrm{d} u
\end{aligned}
$$

which provides a holomorphic extension of $\alpha \mapsto M^{\alpha} \phi(t)$ to $\operatorname{Re} \alpha>-n$. One also verifies from (9.2) that $M^{-(n-1)} \phi(t)=t^{n-1}(\partial / \partial t)^{n-1} \phi(t)$; thus $I^{-(n-1)}=(\partial / \partial t)^{n-1}$ and in particular $I^{0}=M^{0}=\mathrm{id}$, the identity operator.

With this notion at hand, both estimates (9.1) and Theorem 5.1 have (after application of Kahane's inequality and Fubini's theorem) the generic form

$$
\left\|\int_{0}^{\infty} t \frac{\partial}{\partial t}\left[M_{t}^{\alpha} T f\right] \frac{\partial B_{t}}{t^{1 / 2}}\right\|_{L^{p}(P \otimes \mu, X)} \leq C\|f\|_{L^{p}(\mu, X)}
$$

the former with $p=2, X=H$ and $\alpha=0,-1,-2, \ldots$; the latter with $p \in] 1, \infty[, X$ UMD, and $\alpha=1$. Somewhat more knowledge is needed for holomorphic interpolation, and this is provided by the following sequence of lemmata: 
9.4 Lemma. For any Banach space $X$, and $1 \leq p<\infty, \operatorname{Re} \beta>0$ and $\gamma>0$, we have

$$
\begin{aligned}
\left(E \| \int_{0}^{\infty} M^{\beta} \phi(t)\right. & \left.\frac{\mathrm{d} B_{t}}{t^{\gamma / 2}} \|_{X}^{p}\right)^{1 / p} \\
& \leq \frac{\sqrt{\Gamma(\operatorname{Re} \beta) \Gamma(\gamma)}}{|\Gamma(\beta)| \sqrt{\operatorname{Re} \beta \cdot \Gamma(\operatorname{Re} \beta+\gamma)}}\left(E\left\|\int_{0}^{\infty} \phi(t) \frac{\mathrm{d} B_{t}}{t^{\gamma / 2}}\right\|_{X}^{p}\right)^{1 / p} ;
\end{aligned}
$$

more precisely, the existence of the right-hand integral implies the existence of the left-hand one and the stated estimate.

Proof. Once again, we resort to Theorem 2.5:

$$
\begin{aligned}
\int_{0}^{\infty} & \left|\left\langle M^{\beta} \phi(t), x^{\prime}\right\rangle\right|^{2} \frac{\mathrm{d} t}{t^{\gamma}} \\
& \leq \frac{1}{|\Gamma(\beta)|^{2}} \int_{0}^{\infty}\left(\int_{0}^{1}(1-u)^{\operatorname{Re} \beta-1}\left|\left\langle\phi(t u), x^{\prime}\right\rangle\right| \mathrm{d} u\right)^{2} \frac{\mathrm{d} t}{t^{\gamma}} \\
& \leq \frac{1}{|\Gamma(\beta)|^{2}} \int_{0}^{1}(1-u)^{\operatorname{Re} \beta-1} \mathrm{~d} u \times \int_{0}^{1}(1-u)^{\operatorname{Re} \beta-1} \int_{0}^{\infty}\left|\left\langle\phi(t u), x^{\prime}\right\rangle\right|^{2} \frac{\mathrm{d} t}{t^{\gamma}} \mathrm{d} u \\
& =\frac{1}{|\Gamma(\beta)|^{2} \cdot \operatorname{Re} \beta} \int_{0}^{1}(1-u)^{\operatorname{Re} \beta-1} u^{\gamma-1} \mathrm{~d} u \cdot \int_{0}^{\infty}\left|\left\langle\phi(t), x^{\prime}\right\rangle\right|^{2} \frac{\mathrm{d} t}{t^{\gamma}} .
\end{aligned}
$$

9.5 Lemma. If the estimate (9.3) holds in a certain Banach space $X$ with $\alpha=1$, then it holds for all $\operatorname{Re} \alpha>1$.

Proof. The crucial observation is the fact that $t D$ commutes with $M^{\beta}$ for $\operatorname{Re} \beta>0$. In fact,

$$
\begin{aligned}
t D M^{\beta} \phi(t) & =\frac{1}{\Gamma(\beta)} \int_{0}^{1}(1-u)^{\beta-1} t \frac{\partial}{\partial t}[\phi(t u)] \mathrm{d} u \\
& =\frac{1}{\Gamma(\beta)} \int_{0}^{1}(1-u)^{\beta-1} t u \phi^{\prime}(t u) \mathrm{d} u=M^{\beta}(t D \phi)(t) .
\end{aligned}
$$

Thus

$$
\begin{aligned}
t D M^{1+\beta} & =t D t^{-(1+\beta)} I^{\beta} I^{1}=t D t^{-1} M^{\beta} t M^{1}=-t^{-1} M^{\beta} t M^{1}+t^{-1} M^{\beta} t D t M^{1} \\
& =-t^{-1} M^{\beta} t M^{1}+t^{-1} M^{\beta} t M^{1}+t^{-1} M^{\beta} t^{2} D M^{1}=t^{-1} M^{\beta} t^{2} D M^{1} .
\end{aligned}
$$

Using the previous identity, Lemma 9.4 and the assumed estimate, in this order, we get

$$
\begin{gathered}
\left\|\int_{0}^{\infty} t \frac{\partial}{\partial t}\left(M_{t}^{1+\beta} T f\right) \frac{\mathrm{d} B_{t}}{t^{1 / 2}}\right\|_{L^{p}(P \otimes \mu, X)}=\left\|\int_{0}^{\infty} M^{\beta}\left(t^{2} \frac{\partial}{\partial t} M_{t}^{1} T f\right) \frac{\mathrm{d} B_{t}}{t^{3 / 2}}\right\|_{L^{p}(P \times \mu, X)} \\
\leq C\left\|\int_{0}^{\infty} t^{2} \frac{\partial}{\partial t}\left(M_{t}^{1} T f\right) \frac{\mathrm{d} B_{t}}{t^{3 / 2}}\right\|_{L^{p}(P \times \mu, X)} \leq C\|f\|_{L^{p}(\mu, X)} .
\end{gathered}
$$


9.6 Lemma. If the estimate (9.3) holds for $\alpha=0,-1, \ldots,-n$, then it holds for all $\operatorname{Re} \alpha>-n$.

Proof. For $\operatorname{Re} \alpha>-n$, we apply the formula (9.2) to $\phi(t)=T^{t} f$ to get

$$
\begin{aligned}
& t \frac{\partial}{\partial t} M_{t}^{\alpha} T f=\frac{1}{\Gamma(\alpha)} \int_{0}^{1 / 2}(1-u)^{\alpha-1}\left(s \frac{\partial}{\partial s} T^{s} f\right)_{s=t u} \mathrm{~d} u \\
& +\sum_{k=0}^{n-1} \frac{1}{2^{\alpha} \Gamma(\alpha+k+1)}\left(k s^{k} \frac{\partial^{k}}{\partial s^{k}} T^{s} f+2 s^{k+1} \frac{\partial^{k+1}}{\partial s^{k+1}} T^{s} f\right)_{s=t / 2} \\
& +\frac{1}{\Gamma(\alpha+n)} \int_{1 / 2}^{1}(1-u)^{\alpha+n-1}\left(n u^{-n} s^{n} \frac{\partial^{n}}{\partial s^{n}} T^{s} f+u^{-(n+1)} s^{n+1} \frac{\partial^{n+1}}{\partial s^{n+1}} T^{s} f\right)_{s=t u} \mathrm{~d} u
\end{aligned}
$$

By assumption, the stochastic integrals of $t^{n+1} \frac{\partial^{n+1}}{\partial t^{n+1}} T^{t} f$ with respect to $\mathrm{d} B_{t} / t^{1 / 2}$ exist on $] 0, \infty[$. It is easy to see (e.g., by Theorem 2.5) that the norms of these stochastic integrals are invariant under the dilations $t \leftarrow u t$. Finally, the functions $(1-u)^{\alpha-1}$ on $[0,1 / 2]$ and $(1-u)^{\alpha+n-1} u^{-n+j}$ $(j \in\{0,1\})$ on $[1 / 2,1]$ are integrable. From these observations the existence of the stochastic integral $\int_{0}^{\infty} t \frac{\partial}{\partial t} M_{t}^{\alpha} T f \mathrm{~d} B_{t} / t^{1 / 2}$, together with a norm estimate, follows.

9.7 Theorem. Let $T^{t}$ be a symmetric diffusion semigroup on $(M, \mu)$. Let $X$ be a closed subspace of a complex interpolation space $[H, Y]_{\theta}$, where $Y$ is a UMD-space, $H$ is a Hilbert space and $0<\theta<1$. Then the square function estimate (9.3) holds for all $\alpha \in \mathbb{C}$, all $1<p<\infty$ and for all $f \in L^{p}(M, \mu)$, with constant independent of $f$. In particular, we have

$$
c\left\|f-E_{0} f\right\|_{L^{p}(\mu, X)} \leq E\left\|\int_{0}^{\infty} t^{k} \frac{\partial^{k}}{\partial t^{k}} T^{t} f \frac{\mathrm{d} B_{t}}{t^{1 / 2}}\right\|_{L^{p}(\mu, X)} \leq C\|f\|_{L^{p}(\mu, X)}
$$

for all $\left.f \in L^{p}(\mu, X), p \in\right] 1, \infty[$, and $k=1,2, \ldots$, where $0<c<C<\infty$ depend on $X, p$ and $k$.

Proof. It is easily seen that

$$
U(\alpha): f \mapsto \int_{0}^{\infty} t \frac{\partial}{\partial t} M_{t}^{\alpha} T f \frac{\mathrm{d} B_{t}}{t^{1 / 2}}
$$

is a holomorphic family of operators in the sense of Stein, and for any strip of $a_{0} \leq \operatorname{Re} \alpha \leq a_{1}$ of the complex plane, where $\max \left(1, a_{0}\right)<a_{1}$ we know already the estimates

$$
\begin{aligned}
& \left\|U\left(a_{0}+\mathbf{i} t\right) f\right\|_{L^{r}(P \otimes \mu, H)} \leq C\left(a_{0}, t\right)\|f\|_{L^{r}(\mu, H)}, \quad r=2, \\
& \left.\left\|U\left(a_{1}+\mathbf{i} t\right) f\right\|_{L^{q}(P \otimes \mu, Y)} \leq C\left(a_{1}, t\right)\|f\|_{L^{q}(\mu, Y)}, \quad q \in\right] 1, \infty[.
\end{aligned}
$$


An investigation of the above lemmata shows that the growth of $C\left(a_{j}, t\right)$ as $|t| \rightarrow \infty$ is admissible for the use of (a vector-valued extension of) the Stein interpolation theorem [20].

We apply this theorem twice. The second line in (9.9) holds in particular with $Y=H$, and hence

$$
\|U(a+\mathbf{i} t) f\|_{L^{r}(P \otimes \mu, H)} \leq C(a, t)\|f\|_{L^{r}(\mu, H)}
$$

provided that $a=(1-\sigma) a_{0}+\sigma a_{1}$ and $1 / r=(1-\sigma) / 2+\sigma / q$ for some $\sigma \in$ ]0,1[. With appropriate choice of the parameters, we see that the first line in (9.9) continues to hold with any $r \in] 1, \infty[$. Taking $r=q=p$ for a desired $p \in] 1, \infty[$, and interpolating between the estimates in (9.9), we finally get

$$
\|U(a+\mathbf{i} t) f\|_{L^{p}\left(P \otimes \mu,[H, Y]_{\theta}\right)} \leq C(a, t)\|f\|_{L^{p}\left(\mu,[H, Y]_{\theta}\right)}
$$

for $a=(1-\theta) a_{0}+\theta a_{1}$, and obviously any $a+\mathbf{i} t \in \mathbb{C}$ is obtained in this way with a suitable choice of $a_{0}<a<a_{1}$.

As already pointed out, the right side of (9.8) for $X=[H, Y]_{\theta}$ is a specialization of the established estimate to negative integer points. The left side follows by repeating the duality argument given for subordinated semigroups, since $[H, Y]_{\theta}^{\prime}=\left[H^{\prime}, Y^{\prime}\right]_{\theta}$ for reflexive spaces $H$ and $Y$, and $H^{\prime}$ and $Y^{\prime}$ are again a Hilbert space and a UMD space.

Finally, if $X$ is only a closed subspace of $[H, Y]_{\theta}$, these estimates still hold in $X$ (with the inherited norm), since the tensor-product type operator $U(\alpha)$ maps $X$-valued functions into $X$-valued ones, so this is just a specialization to of the proved estimate to those $f \in L^{p}\left(\mu,[H, Y]_{\theta}\right)$ which take their values in $X$.

9.10 Corollary. Let $X$ be isomorphic to a closed subspace of a complex interpolation space $[H, Y]_{\theta}$ as in Theorem 9.7, let $\left.p \in\right] 1, \infty\left[\right.$, and $T^{t}$ be a symmetric diffusion semigroup on some $(M, \mu)$. Then for all functions $a:[0, \infty[\rightarrow \mathscr{L}(X)$ as in Theorem 1.9 , or more generally Prop. 7.4 , the operator $T_{a}$ from (1.2) defines a bounded mapping on $L^{p}(\mu, X)$, with norm estimated by

$$
\left\|T_{a}\right\|_{\mathscr{L}\left(L^{p}(\mu, X)\right)} \leq C \mathscr{R}(\mathscr{T}) \sup _{0<T<\infty} T^{-1 / 2}\|\| a(t)\left\|_{\mathscr{T}}\right\|_{L^{2}(0, T)} \leq C \mathscr{R}(a),
$$

where $C$ depends only on $X$ and $p$. In fact, the negative generator $A$ of $T^{t}=e^{-t A}$ has a bounded $H^{\infty}\left(\Sigma_{(1-\theta)|1 / p-1 / 2| \pi+\theta \pi / 2+\epsilon}\right)$ functional calculus for every $\epsilon>0$.

Proof. This multiplier estimate follows from Theorem 9.7 in the same way as Theorem 1.6 implied Theorem 1.9, and its generalization in $\S 7$. 
As in $\S 8$ for subordinated semigroups, the estimate for $T_{a}$ implies that the negative generator $A$ of $T^{t}=e^{-t A}$ has a bounded $H^{\infty}\left(\Sigma_{\pi / 2}\right)$ functional calculus on $L^{p}(\mu, X)$. The angle may be improved by a further interpolation argument:

First, if $H$ is a Hilbert space, then $A$ has the Borel functional calculus (i.e., angle 0$)$ on $L^{2}(\mu, H)$ by spectral theory and an $H^{\infty}\left(\Sigma_{\pi / 2}\right)$ calculus on $L^{q}(\mu, H)$ for $\left.q \in\right] 1, \infty[$. If $1 / p=(1-\alpha) / 2+\alpha / q$, we obtain by interpolation a bounded $H^{\infty}\left(\Sigma_{(1-\alpha) \cdot 0+\alpha \pi / 2}\right)$ calculus on $L^{p}(\mu, H)$. Taking $q \rightarrow 1$ or $q \rightarrow \infty$ according to $p<2$ or $p>2$, we find that $A$ has a bounded $H^{\infty}\left(\Sigma_{|1 / p-1 / 2| \pi+\epsilon}\right)$ calculus on $L^{p}(\mu, H)$ for every $\epsilon>0$.

Next, we observe that $[H, Y]_{\theta}=\left[H,[H, Y]_{\beta}\right]_{\theta / \beta}$ by the re-iteration theorem for $0<\theta<\beta<1$. An interpolation argument between the $H^{\infty}\left(\Sigma_{|1 / p-1 / 2| \pi+\epsilon}\right)$ calculus on $L^{p}(\mu, H)$ and the $H^{\infty}\left(\Sigma_{\pi / 2}\right)$ calculus on $L^{p}\left(\mu,[H, Y]_{\beta}\right)$ gives an $H^{\infty}\left(\Sigma_{(1-\theta / \beta)(|1 / p-1 / 2| \pi+\epsilon)+\theta \pi / 2 \beta}\right)$ calculus on $L^{p}\left(\mu,[H, Y]_{\theta}\right)$. Taking $\beta \rightarrow 1$, we obtain the desired conclusion.

\section{References}

[1] Berkson, E. And Gillespie, T. A.: Spectral decompositions and harmonic analysis on UMD spaces. Studia Math. 112 (1994), no. 1, 13-49.

[2] Berkson, E. And Gillespie, T. A.: An $\mathfrak{M}_{q}(\mathbb{T})$-functional calculus for power-bounded operators on certain UMD spaces. Studia Math. 167 (2005), no. 3, 245-257.

[3] Bourgain, J.: Some remarks on Banach spaces in which martingale difference sequences are unconditional. Ark. Mat. 21 (1983), no. 2, 163-168.

[4] Bourgain, J.: Vector-valued singular integrals and the $H^{1}$-BMO duality. In Probability theory and harmonic analysis (Cleveland, Ohio, 1983), 1-19. Monogr. Textbooks Pure Appl. Math. 98. Marcel Dekker, New York, 1986.

[5] Clément, P., De Pagter, B., Sukochev, F. A. and Witvliet, H.: Schauder decomposition and multiplier theorems. Studia Math. 138 (2000), no. $2,135-163$.

[6] Clément, P. And Prüss, J.: Some remarks on maximal regularity of parabolic problems. In Evolution equations: applications to physics, industry, life sciences and economics (Levico Terme, 2000), 101-111. Progr. Nonlinear Differential Equations Appl. 55. Birkhäuser, Basel, 2003.

[7] Cowling, M., Doust, I., McIntosh, A. And Yagi, A.: Banach space operators with a bounded $H^{\infty}$ functional calculus. J. Austral. Math. Soc. Ser. A 60 (1996), no. 1, 51-89.

[8] Diestel, J., Jarchow, H. And Tonge, A.: Absolutely summing operators. Cambridge Studies in Advanced Math. 43. Cambridge Univ. Press, Cambridge, 1995.

[9] Dodds, P. G., Dodds, T. K. And de Pagter, B.: Fully symmetric operator spaces. Integral Equations Operator Theory 15 (1992), no. 6, 942-972. 
[10] Doob, J. L.: A ratio operator limit theorem. Z. Wahrscheinlichkeitstheorie und Verw. Gebiete 1 (1962/1963), 288-294.

[11] Guerre-Delabrière, S.: Some remarks on complex powers of $(-\Delta)$ and UMD spaces. Illinois J. Math. 35 (1991), no. 3, 401-407.

[12] Kalton, N. And Weis, L.: The $H^{\infty}$-functional calculus and square function estimates. Manuscript in preparation.

[13] Kunstmann, P. C. And Weis, L.: Maximal $L_{p}$-regularity for parabolic equations, Fourier multiplier theorems and $H^{\infty}$-functional calculus. In Functional analytic methods for evolution equations, 65-311. Lecture Notes in Math., 1855. Springer, Berlin, 2004.

[14] Martínez, T., Torrea, J. L. And Xu, Q.: Vector-valued LittlewoodPaley-Stein theory for semigroups. Adv. Math. 203 (2006), no. 2, 430-475.

[15] van Neerven, J. M. A. M., Veraar, M. C. and Weis, L.: Stochastic integration in UMD Banach spaces. Ann. Probab. 35 (2007), 1438-1478.

[16] van Neerven, J. M. A. M. And Weis, L.: Stochastic integration of functions with values in a Banach space. Studia Math. 166 (2005), 133-170.

[17] Rosiński, J. And Suchanecki, Z.: On the space of vector-valued functions integrable with respect to the white noise. Colloq. Math. 43 (1980), no. $1,183-201$.

[18] Rota, G.-C.: An "alternierende Verfahren" for general positive operators. Bull. Amer. Math. Soc. 68 (1962), 95-102.

[19] Rubio De Francia, J. L.: Martingale and integral transforms of Banach space valued functions. In Probability in Banach spaces (Zaragoza, 1985), 195 - 222. Lecture Notes in Math. 1221. Springer, Berlin, 1986.

[20] Stein, E. M.: Interpolation of linear operators. Trans. Amer. Math. Soc. 83 (1956), 482-492.

[21] Stein, E. M.: Topics in harmonic analysis related to Littlewood-Paley theory. Annals of Mathematics Studies 63. Princeton University Press, Princeton, N.J.; University of Tokyo Press, Tokyo, 1970.

[22] WeIs, L.: Operator-valued Fourier multiplier theorems and maximal $L_{p^{-}}$ regularity. Math. Ann. 319 (2001), no. 4, 735-758.

[23] XU, Q.: Littlewood-Paley theory for functions with values in uniformly convex spaces. J. Reine Angew. Math. 504 (1998), 195-226.

Recibido: 11 de abril de 2006

Tuomas P. Hytönen Department of Mathematics and Statistics

University of Helsinki

Gustaf Hällströmin katu 2b

FI-00014 Helsinki, Finland tuomas.hytonen@helsinki.fi 